\title{
LAS CONSONANTES [-r] Y [-1] IMPLOSIVAS EN ANDALUCÍA
}

\author{
MARÍA JOSÉ QUILIS-SANZ
}

\section{INTRODUCCIÓN}

Al examinar la bibliografía sobre la neutralización y el comportamiento, en general, de las consonantes [-r] y [-1] implosivas en Andalucía, nos dimos cuenta de que el fenómeno había sido estudiado, en artículos o monografias, en determinadas zonas, dejando la mayor parte de la mencionada región sin examinar. En nuestra opinión, hacía falta un estudio global de este fenómeno que, aunque complejo, era factible, existiendo los ricos materiales contenidos en el Atlas Lingüístico y Etnográfico de Andalucía. Ya Amado Alonso y Raimundo Lida señalaron la carencia de visión de conjunto en la investigación del mencionado fenómeno cuando decían: «todos los dialectólogos dan noticia de ello, aunque ninguno deslinda áreas de igualación, ni podemos nosotros emprender esta tarea reuniendo y combinando los datos de todos ellos. Tampoco suelen ser explícitos los dialectólogos en fijar las condiciones fonéticas de la igualación de ambos fonemas, ya en $r$ ya en $l{ }^{1}$. Y esto es lo que hemos pretendido hacer modestamente en nuestro estudio sobre el territorio andaluz ${ }^{2}$.

\footnotetext{
1 Amado Alonso y Raimundo Lida, «Geografia fonética: $-l$ y $-r$ implosivas en español», Revista de Filología Hispánica, VII, 1945, págs 313-345. Recogido en A. Alonso, Estudios lingüisticos. Temas hispanoamericanos, Madrid, Gredos, 1953, págs 213-267. Citamos por esta última edición. La referencia mencionada está en la pág. 219.

${ }^{2}$ Los trabajos donde con más extensión ha sido tratada esta cuestión en la región andaluza son los siguientes: Amado Alonso y Raimundo Lida, op. cit., págs. 219-220. L. Rodríguez Castellano y Adela Palacio, «El habla de Cabra», RDTP, IV, 1948, págs. 387-418 y 570-599. Gregorio Salvador, «El habla de Cúllar-Baza», RFE, XLI, 1957, págs 161-252. Manuel Alvar, «Sevilla, macrocosmos lingüístico. Fonética y fonología según el Atlas Lingüístico y Etnográfico de Andalucía», Estudios filológicos y lingüísticos, Homenaje a Ángel Rosenblat en sus setenta años. Caracas, 1974, págs. 13-42, y «Modalidades fonéticas cordobesas en el Atlas Lingüístico y Etnográfico de Andalucía», Revista de Estudios Regionales, III, 1981, págs. 71-91. Manuel Ariza, «El cambio -R>-L en la provincia de Málaga», Jábega, 1974, págs. 60-62.
} 


\section{NUESTRO ESTUDiO}

Comenzamos trabajando con los ricos materiales contenidos en el Atlas Lingüiśtico y Etnográfico de Andalucia $(A L E A)^{3}$. En él, buscamos los mapas con palabras que tuviesen [-r] y [-1] postnucleares seguidas de pausa o de otra consonante. En cada mapa, examinamos la realización de las mencionadas consonantes en todas las provincias, y dimos los porcentajes correspondientes.

En total, estudiamos 83 mapas del $A L E A$. El número de casos examinados asciende a 16.909 .

Cuando habíamos acabado de elaborar los datos del $A L E A$, apareció la obra Textos andaluces en transcripción fonética, de Manuel Alvar, Antonio Llorente y Gregorio Salvador ${ }^{4}$. Hemos hecho el recuento de las realizaciones de [-r] y [-1] de todo el libro. Pese a ello, el número de casos que hemos recogido - 2.033 - es muy inferior al del ALEA: son las restricciones lógicas que la lengua hablada impone a la fonotáctica del decurso ${ }^{5}$. Debemos advertir que en estos Textos hemos tenido en cuenta, y hemos estudiado por separado, los siguientes casos: a) cuando las mencionadas consonantes líquidas se encontraban ante pausa; $b$ ) cuando se encontraban en interior de palabra ante un segmento determinado, como [rp] en torpe; c) cuando se encontraban en posición final de palabra ante el mismo segmento, como en dar patadas.

Los contornos estudiados de [-r] y [-1] han sido los siguientes:

Juan Antonio Moya Corral, La pronunciación del español en Jaén, Universidad de Granada, 1979, págs. 99-111. Rafael Cano Aguilar y Manuel Cubero Urbano, «Apuntes sobre el habla de Osuna», Archivo Hispalense, LXII, 189 (1979), págs. 17-40. Josefa Mendoza Abreu, Contribución al estudio del habla rural y marinera de Lepe (Huelva), Excma. Diputación Provincial de Huelva, 1985. Pilar Carrasco Campos, Contribución al estudio del habla rural de Baeza (Jaén), Instituto de Estudios Gienenses, C.S.I.C., Jaén, 1981, págs. 71-76. José María Becerra Hiraldo y Cándida Vargas Labella, Aproximación al español hablado en Jaén, Granada, Universidad de Granada, 1986. Pedro Payán Sotomayor, La pronunciación del español en Cádiz, Cádiz, Universidad de Cádiz, 1988. Manuel Galeote, El habla rural del Treviño de Iznájar, Villanueva de Tapia y Venta de Santa Bárbara, Granada, Ediciones TAT, 1988. Pedro Carbonero Cano, José Luis Álvarez Cubero, Joaquín Casas Gómez, Isabel M. Gutiérrez Sánchez, El habla de Jerez. Estudio sociolingüístico, BUC, Cuadernos de divulgación, 5, s. 1., s.a. [1992]. Para datos similares, procedentes de Toledo y Ciudad Real, véase Pilar García Mouton y Francisco Moreno Fernández, «El Atlas Lingüístico y Etnográfico de Castilla-La Mancha. Materiales fonéticos de Ciudad Real y Toledo», Geolingüística. Trabajos europeos, ed. por P. García Mouton, Madrid, C.S.I.C., 1994, págs. 141-147.

${ }^{3}$ Obra debida a Manuel Alvar, Antonio Llorente Maldonado de Guevara y Gregorio Salvador, 6 vols., Granada, 1960-1973.

${ }^{4}$ Edición de Manuel Alvar y Pilar García Mouton, Madrid, Gredos, 1995. En adelante, nos referiremos a esta obra como Textos.

5 Piénsese, por ejemplo, que en la lengua hablada, $/ \mathrm{R} /$, que es el equivalente de la [-r] que aquí nos ocupa, tiene una frecuencia del $1,93 \%$, a lo que hay que añadir las restricciones de la combinatoria que estudiamos (Vid. Quilis, Tratado de Fonología y Fonética españolas, Madrid, Gredos, 1993, pág. 43). 


\section{1. [-r] final de palabra ante pausa}

2.1.1. En el ALEA. Número de mapas: 12: uncir (mapa n. $\left.{ }^{\circ} 134\right)$; decir (mapa n..$^{\circ} 1624$ ); coger (mapa n. ${ }^{\circ} 1286$ ); llover (mapa n..$^{\circ} 848$ ); morder (mapa n. ${ }^{\circ} 1222$ ); anteayer (mapa n. ${ }^{\circ} 821$ ); alfiler (mapa $n^{\circ}$ 1746); saltar (mapa n. ${ }^{\circ}$ 1422); eructar (mapa n. ${ }^{\circ} 1240$ ); guiñar (mapa n. $\left.{ }^{\circ} 1197\right)$; escozor (mapa n..$^{\circ} 1198$ ); flor (mapa n. ${ }^{\circ} 1544$ ).

El número de casos estudiados asciende a 2.446 . El porcentaje de las distintas realizaciones para cada provincia están señaladas en el cuadro siguiente ${ }^{6}$ :

\begin{tabular}{lrrllllll}
\hline & $-\mathrm{r}$ & -1 & $\mathrm{l}$ & $\mathrm{v}:$ & $\mathrm{v}$ & $\mathrm{v}$ & $\mathrm{h}$ & $\mathrm{n}$ \\
\hline $\mathrm{Al}$ & 18,9 & 51,8 & 2,1 & 10,8 & 4 & 12,1 & & \\
$\mathrm{Gr}$ & 16,6 & 32,7 & 1,8 & 21,5 & 7,7 & 17,2 & 2,2 & \\
$\mathrm{~J}$ & 18,7 & 30 & 1 & 15,7 & 15,1 & 18,7 & 0,5 & \\
$\mathrm{Co}$ & 16,1 & 23,2 & 3,2 & 27,2 & 12,1 & 16,4 & 0,7 & 0,7 \\
$\mathrm{Ma}$ & 3,6 & 3,2 & 0,3 & 38,4 & 34,7 & 19,5 & & \\
$\mathrm{Se}$ & 1,5 & 0,9 & & 41,2 & 43,7 & 11,1 & & 1,2 \\
$\mathrm{Ca}$ & 1,9 & 0,4 & & 20 & 58,3 & 18,1 & & 1 \\
$\mathrm{Hu}$ & 2,1 & 14,9 & & 22,2 & 52,5 & 7,6 & & 0,4 \\
$\mathrm{And}$ & 11,3 & 22,1 & 1,1 & 24,1 & 24,7 & 15,4 & 0,5 & 0,3 \\
\hline
\end{tabular}

1. Al examinar los datos no hemos apreciado que el tipo de vocal que precede a [-r] influya en el mantenimiento o pérdida de esta articulación.

2. Como puede observarse en el cuadro que ofrecemos, las provincias de $\mathrm{Ma}, \mathrm{Se}, \mathrm{Ca}$ y $\mathrm{Hu}$, o sea, la llamada Andalucía occidental, son las que presentan el mayor porcentaje de pérdida de la consonante.

3. En los resultados generales que aparecen en el cuadro, puede observarse que en el total de Andalucía, el número de casos de pérdida de la consonante $[-r](64,2 \%)$ supera al número de casos de mantenimiento de un segmento consonántico.

4. Cuando el segmento consonántico se mantiene, la realización que predomina, con mucho, es [-1] $(22,1 \%)$, seguida de [-r] $(11,3 \%)$ y, a mucha distancia, $\left[\begin{array}{l}1 \\ 1\end{array}\right]$.

5. En las provincias de $\mathrm{Al}, \mathrm{Gr}, \mathrm{J}, \mathrm{Co}$, el mantenimiento del segmento consonántico supone el 53,9\% de los casos. Aquí, también la realización mayoritaria es $[-1](23,2 \%)$, seguida de $[-r](16,1 \%) \mathrm{y}$, a mucha distancia, $\left[\begin{array}{l}1 \\ 1\end{array}\right]$.

6. En las palabras terminadas en -er, hay verbos y otras partes de la oración, y debemos advertir que entre ambos grupos de palabras no hay di-

\footnotetext{
${ }^{6}$ En los cuadros que damos a continuación, $\left[\begin{array}{l}1 \\ 1\end{array}\right]$ representa la realización intermedia entre las dos líquidas. [v:] representa un vocal cualquiera, larga; [v], una vocal normal, y [v'] una vocal abierta; [vn], una vocal nasal; todas ellas quedan como núcleo silábico de una sílaba abierta, al perderse la consonante líquida que la seguía.
} 
ferencias en cuanto al comportamiento de la mencionada consonante vibrante.

2.1.2. En los Textos. El número de casos aparecidos es, como ya indicamos antes, muchísimo menor: 125. Los porcentajes pueden verse en el cuadro siguiente:

\begin{tabular}{lcccc}
\hline & $-r$ & -1 & $\emptyset$ & ) \\
\hline $\mathrm{Al}$ & & 87,5 & 12,5 & \\
$\mathrm{Gr}$ & 27 & 9 & 64 & \\
$\mathrm{~J}$ & 36 & 28 & 36 & \\
$\mathrm{Co}$ & 7 & 36 & 57 & 4,5 \\
$\mathrm{Ma}$ & 4,5 & & 91 & \\
$\mathrm{Se}$ & & & 100 & \\
$\mathrm{Ca}$ & & & 100 & 0,8 \\
$\mathrm{Hu}$ & 8 & 13,6 & 100 & 77,6 \\
And. & & & &
\end{tabular}

Como en el $A L E A$, las provincias de $\mathrm{Ma}, \mathrm{Se}, \mathrm{Ca}$ y $\mathrm{Hu}$ son las que presentan el mayor porcentaje de pérdida de esta consonante.

En el total de Andalucía, esta pérdida supone un porcentaje muy alto: el $77,6 \%$.

Cuando se mantiene un segmento consonántico, predomina la realización [-1] $(13,6 \%)$ sobre $[-r](8 \%)$.

\section{2. [-l] final de palabra ante pausa}

2.2.1. En el ALEA. Número de mapas: 11: perejil (mapa n. ${ }^{\circ}$ 1614); miel (mapa n. ${ }^{\circ} 1533$ ); hiel (mapa n. ${ }^{\circ} 1549$ ); corral (mapa n. ${ }^{\circ}$ 678); carnaval (mapa n..$^{\circ}$ 1476); pedernal (mapa n. ${ }^{\circ}$ 804); umbral (mapa n. ${ }^{\circ}$ 662); farol (mapa n. ${ }^{\circ} 1543$ ); árbol (mapa n. $\left.{ }^{\circ} 1616\right)$; sol (mapa n. $\left.{ }^{\circ} 1618\right)$; azul (mapa n. ${ }^{\circ} 1619$ ). El número de casos estudiados es de 2.290. Las soluciones aparecen en el siguiente cuadro:

\begin{tabular}{lcccccccccc}
\hline & -1 & $-\mathrm{r}$ & $\mathrm{l}$ & $\mathrm{v}:$ & \multicolumn{1}{c}{$\mathrm{v}$} & $\mathrm{v}$ & $\mathrm{vn}$ & $\mathrm{h}$ & $\mathrm{s}$ & $\mathrm{n}$ \\
\hline $\mathrm{Al}$ & 56,9 & 2,4 & 1,7 & 5,2 & 4,1 & 28,4 & 0,3 & 0,3 & & 0,3 \\
$\mathrm{Gr}$ & 39,9 & 2,4 & 1,1 & 12,1 & 6 & 36,5 & & 1,7 & & \\
$\mathrm{~J}$ & 45,8 & 2,8 & 0,3 & 10,5 & 4,8 & 35,2 & & 0,3 & & \\
$\mathrm{Co}$ & 32,7 & 2,2 & 1,8 & 16,9 & 9,7 & 35,2 & 1,1 & & & \\
$\mathrm{Ma}$ & 7,6 & 0,7 & 0,3 & 34,7 & 21,9 & 29,6 & 2,9 & 0,3 & & 1,4 \\
$\mathrm{Se}$ & 4,7 & 1 & & 41,9 & 26,6 & 19,7 & 3 & 0,3 & 0,3 & 2 \\
$\mathrm{Ca}$ & 3,4 & & & 34 & 52,2 & 9 & & & & 1,1 \\
$\mathrm{Hu}$ & 13,5 & & 2,1 & 34,7 & 30,5 & 14,8 & 2,5 & 0,4 & & 1,2 \\
And. & 28,1 & 1,6 & 0,9 & 22,1 & 16,6 & 27,8 & 1,1 & 0,5 & 0,04 & 0,6 \\
\hline
\end{tabular}


1. Como en el caso de [-r], no hemos notado que el tipo de vocal que precede a esta consonante lateral haya influido en su mantenimiento, pérdida o modificación.

2. Según los datos que ofrece el mencionado cuadro, las provincias de $\mathrm{Ma}, \mathrm{Se}, \mathrm{Ca}$ y $\mathrm{Hu}$ son, nuevamente, las que presentan el porcentaje más alto de pérdida de la consonante.

3. En el total de Andalucía, el porcentaje de casos de pérdida de [-1] (67,6\%, cifra semejante a la de la pérdida de la vibrante) supera al número de casos de mantenimiento de un segmento consonántico. Cuando éste se mantiene, la realización que predomina con mucho es [-1] $(28,1 \%$, cifra algo superior a la de la realización de la vibrante, pero en ambos casos, la más alta), seguida a mucha distancia de $[-r](1,6 \%)$ y $\left[\begin{array}{l}1 \\ I\end{array}\right](0,9 \%)$.

4. En las provincias de $\mathrm{Ma}, \mathrm{Se}, \mathrm{Ca}, \mathrm{Hu}$, en las que, como hemos dicho, es más alta la frecuencia de pérdida de la consonante, su vocal núcleo silábico se realiza la mayoría de las veces como vocal larga. Lo contrario sucede en las provincias de $\mathrm{Al}, \mathrm{Gr}$, J, donde es menor la pérdida de la consonante $\mathrm{y}$, donde, a su vez, la realización de la vocal núcleo silábico es mayoritariamente abierta.

5. En las provincias de $\mathrm{Al}, \mathrm{Gr}, \mathrm{J}$ y $\mathrm{Co}$, la pérdida de la consonante también es mayor que su mantenimiento, aunque la diferencia es muy pequeña: mantenimiento de la consonante: $48,1 \%$; pérdida $51,8 \%$. En estas provincias, la realización mayoritaria es [-1], con el $43,6 \% \mathrm{y}$, a mucha distancia, [-r] $(2,5 \%),\left[\begin{array}{l}1 \\ I\end{array}(1,2 \%),[\mathrm{h}](0,7 \%), \mathrm{y}[\mathrm{n}](0,07 \%)\right.$.

2.2.2. En los Textos. El número de casos recogidos asciende a $46 . \mathrm{Su}$ distribución aparece en el siguiente cuadro:

\begin{tabular}{lccc}
\hline & $-r$ & -1 & $\varnothing$ \\
\hline $\mathrm{Al}$ & & 50 & 50 \\
$\mathrm{Gr}$ & 33,3 & 66,6 & \\
$\mathrm{~J}$ & & 28,5 & 71,4 \\
$\mathrm{Co}$ & 20 & 33,3 & 66,6 \\
$\mathrm{Ma}$ & & 50 & 60 \\
$\mathrm{Se}$ & & & 94,2 \\
$\mathrm{Ca}$ & & 100 & 100 \\
$\mathrm{Hu}$ & 4,4 & 19,5 & 76 \\
$\mathrm{And}$. & & & \\
\hline
\end{tabular}

El mayor porcentaje de pérdida de esta consonante se produce en las provincias de $\mathrm{Se}, \mathrm{Ca}$ y $\mathrm{Hu}$.

En el total de Andalucía, la pérdida de [-1] supone un $76 \%$. Se mantiene en un $19,5 \%$ y se realiza como [-r] en un $4,4 \%$. 


\subsection{Secuencia heterosilábica [-rp-]}

2.3.1. En el ALEA. Número de mapas estudiados: 2: carpintero (mapa n. ${ }^{\circ}$ 935) y párpado (mapa n. ${ }^{\circ} 1200$ ). El número de casos estudiados asciende a 345 .

\begin{tabular}{llllll}
\hline & -rp- & -lp- & $-\frac{1}{1} \mathrm{p}-$ & $-\mathrm{mp}-$ & -hp- \\
\hline $\mathrm{Al}$ & 86 & 7 & & 7 & \\
$\mathrm{Gr}$ & 90 & 3,2 & 3,2 & 3,2 & \\
$\mathrm{~J}$ & 85 & 8,8 & 5,8 & & \\
$\mathrm{Co}$ & 95,6 & 2,1 & 2,1 & & \\
$\mathrm{Ma}$ & 87 & 10,9 & 2,1 & & 3 \\
$\mathrm{Se}$ & 100 & & & & \\
$\mathrm{Ca}$ & 97 & 3,5 & & & 0,3 \\
$\mathrm{Hu}$ & 96,5 & 4,3 & 1,7 & 1,5 & \\
And. & 92 & & & & \\
\hline
\end{tabular}

1. Según muestra el cuadro anterior, en ningún caso se ha perdido la vibrante implosiva: siempre aparece algún segmento consonántico como realización de ella.

2. Con un porcentaje del $92 \%$, la vibrante se mantiene como tal en toda Andalucía. En el caso de Sevilla, siempre es [-r]. A mucha distancia aparece pronunciada como [-1] implosiva, con un porcentaje del 4,3\%.

3. Con porcentajes muy pequeños aparecen las realizaciones $\left[{ }_{x}^{1} \mathrm{p}\right]$, con un $1,7 \%$, [mp], con un $1,5 \%$ y [hp], con un $0,3 \%$.

2.3.2. En los Textos. El número de casos es muy pequeño: 24: en interior de palabra, 5; en final de palabra: 19.

Los resultados son los siguientes: en interior de palabra, siempre se ha conservado [r]; en final de palabra, se ha mantenido en el $26 \%$ de los casos; se ha perdido en el $53 \%$, y se ha realizado como [1] en el $16 \%$. Otras realizaciones comprenden el restante porcentaje.

\subsection{Secuencia heterosilábica $[-r b-]^{7}$}

2.4.1. En el $A L E A$. Número de mapas: 3: berbiquí (mapa n. ${ }^{\circ}$ 937), corva (mapa n. ${ }^{\circ}$ 1290), barbecho (mapa n. ${ }^{\circ} 21$ ). El número de casos estudiados es de 625 .

\footnotetext{
${ }^{7}$ En las cabeceras de los cuadros, para simplificar, no distinguiremos realizaciones fricativas u oclusivas de $/ \mathrm{b} /, / \mathrm{d} /, / \mathrm{g} /$.
} 


\begin{tabular}{lcccccc}
\hline & - rb- & - lb- & $-\frac{1}{I} \mathrm{~b}-$ & $-\varnothing b-$ & -hb- & -rØ- \\
\hline $\mathrm{Al}$ & 92,2 & 5,5 & 2,2 & & & \\
$\mathrm{Gr}$ & 85,1 & 4,4 & 7,4 & 2,9 & & \\
$\mathrm{~J}$ & 88,8 & 8,8 & 1,1 & 1,1 & & \\
$\mathrm{Co}$ & 81,3 & 5,2 & 5,2 & 6,5 & 1,3 & \\
$\mathrm{Ma}$ & 80,7 & 11,4 & 1,2 & 5,1 & 1,2 & \\
$\mathrm{Se}$ & 80,2 & & 1,2 & 16 & & 2,4 \\
$\mathrm{Ca}$ & 96,7 & & & 3,2 & & \\
$\mathrm{Hu}$ & 97,7 & & & 2,2 & & \\
And. & 86,5 & 5 & 3 & 4,5 & 0,3 & 0,3 \\
\hline
\end{tabular}

1. A diferencia de lo que ocurría en la secuencia [-rp-], en la que ahora estudiamos, aparecen algunos casos de pérdida de la vibrante implosiva. Su porcentaje en el total de Andalucía no es muy grande: el 4,5\%.

2. También en esta secuencia predomina el mantenimiento de [-r]; su porcentaje es el más alto: $86,5 \%$.

3. Sigue, a mucha distancia, su articulación como lateral, con un $5 \%, y$ como la mixta $\left[{ }_{I}^{1} b\right]$, con un $3 \%$.

4. Por último, y con un porcentaje muy pequeño - del $0,3 \%$ - se produce la realización de la vibrante como una aspiración y, por otro lado, la pérdida de la bilabial sonora ${ }^{8}$.

5. Hemos de señalar que han aparecido algunos casos de realizaciones [rb] y [lb], donde el fonema /b/ se ha realizado como oclusivo, en una distribución semejante a la que aparece en algunos lugares de Hispanoamérica ${ }^{9}$ : se trata de los puntos Co 202, Gr 511 y Ma 300, para berbiquí.

2.4.2. En los Textos. En interior de palabra, 84 casos; en final de palabra, 13. Los resultados son los siguientes:

1. Cuando [-r] se encuentra en interior de palabra, no se pierde: se mantiene como tal en el $89 \%$ de los casos, y se realiza como [1] en el resto.

2. Cuando [-r] se encuentra en final de palabra, se mantiene en el $38,5 \%$ de los casos, se pierde en un porcentaje idéntico, se realiza como [1] en un $15 \%$, y como $\left[\begin{array}{l}1 \\ 1\end{array}\right]$, en el resto.

3. También en estos materiales se ha realizado [b] como oclusiva en Ma 407: [bolbé] volver, y [álbo] árbol, tres veces, en H 201.

\subsection{Secuencia heterosilábica [-lp-]}

2.5.1. En el $A L E A$. Número de mapas: 2: pulpejo (mapa n. $^{\circ} 1275$ ) y alpargata (mapa $\mathrm{n}^{\circ}$ 1407). Total de casos: 449.

${ }^{8}$ Indicado por [rØ] en el cuadro.

9 Vid. Antonio Quilis, op. cit., pág. 221. 


\begin{tabular}{lllllll}
\hline & -lp- & -rp- & $-{ }_{1}^{1}$ p- & $-\varnothing p-$ & -hp- & -pp- \\
\hline $\mathrm{Al}$ & 22 & 34 & 2 & 39 & & \\
$\mathrm{Gr}$ & 11 & 29 & 11 & 48 & 1 & \\
$\mathrm{~J}$ & 18 & 20 & 18 & 43 & & \\
$\mathrm{Co}$ & 18,4 & 49 & 10 & 22,5 & & \\
$\mathrm{Ma}$ & 11,5 & 31 & 7,5 & 46 & 2 & 2 \\
$\mathrm{Se}$ & 13 & 45 & 6,5 & 34 & 1,5 & \\
$\mathrm{Ca}$ & 3,3 & 56,5 & & 40 & & \\
$\mathrm{Hu}$ & 27,6 & 32 & 6,4 & 34 & & \\
$\mathrm{And}$ & 16 & 35 & 9 & 39 & 0,7 & 0,2 \\
\hline
\end{tabular}

1. Al estudiar el cuadro correspondiente, se observa que el panorama ha cambiado en comparación con los datos que ofrecíamos en los dos epígrafes anteriores. Aquí, la realización de [-1] que acapara el mayor porcentaje, es la de la pérdida de la lateral, con un $39 \%$. El timbre de la vocal núcleo silábico no se ve afectado por ello.

2. En el rango de frecuencias, sigue la realización [rp], con un $35 \%$.

3. El mantenimiento de [1] en [lp] alcanza sólo el $16 \%$, y en la realización mixta $\left[{ }_{1}^{1} \mathrm{p}\right]$, el $9 \%$.

4. Con porcentajes muy pequeños, aparecen [hp], con un $0,7 \%$ y [pp] con un $0,2 \%$.

2.5.2. En los Textos. El total de casos asciende a 65: en interior de palabra, 4, y en final, 61. Los resultados son los siguientes:

1. En interior de palabra, [-1] se realiza como [-r] en el $75 \%$ de los casos, y como [-1], en el $25 \%$.

2. En posición final de palabra, [-1] se mantiene en el $47,5 \%$ de los casos; se pronuncia como [-r] en el $41 \%$; se pierde en el $9,8 \%$, y es $\left[\begin{array}{l}1 \\ 1\end{array}\right]$ en el resto.

\subsection{Secuencia heterosilábica [-lb-]}

2.6.1. En el ALEA. Número de mapas: 3: albaricoque (mapa n. ${ }^{\circ} 353$ ), que presenta formas lexicalizadas en las que se ha perdido la lateral, sobre todo en las provincias de $\mathrm{Al}, \mathrm{Gr}, \mathrm{Ma}$; albahaca (mapa $\mathrm{n}^{\circ}$ 297), polvareda (mapa n. $^{\circ}$ 1588). El número de casos es de 641.

\begin{tabular}{llllll}
\hline & $-\mathrm{lb}-$ & $-\mathrm{rb}-$ & $-\frac{1}{\mathrm{~d}} \mathrm{~b}-$ & $-\varnothing \mathrm{b}-$ & $-\mathrm{hb}-$ \\
\hline $\mathrm{Al}$ & 28,5 & 30,4 & 14,2 & 26,3 & \\
$\mathrm{Gr}$ & 28 & 32 & 15,6 & 24 & \\
$\mathrm{~J}$ & 54,8 & 28 & 15 & 2 & \\
$\mathrm{Co}$ & 34,6 & 44 & 20 & 1,3 & 1 \\
$\mathrm{Ma}$ & 25 & 32 & 11 & 31 & \\
$\mathrm{Se}$ & 16 & 61,5 & 13 & 9 & \\
$\mathrm{Ca}$ & 8 & 78 & 8 & 5,5 & \\
$\mathrm{Hu}$ & 42 & 45 & 7 & 5,5 & 0,1 \\
And. & 31 & 40 & 13,7 & 14,9 & \\
\hline
\end{tabular}


1. También en este caso el número de realizaciones de la lateral como [-r] es muy elevado: el $40 \%$.

2. Sigue el mantenimiento de [-1], con un $31 \%$.

3. Con porcentajes muy parecidos, aparecen la realización mixta $\left[\begin{array}{l}1 \\ 1\end{array}\right]$, con un $13,75 \%$, y su pérdida con un $14,95 \%$.

4. Sólo ha aparecido un caso de realización como aspirada, [h], en Ma, lo que supone un $0,1 \%$.

5. Como en el caso de [rb], también hemos encontrado puntos donde la realización de /b/ después de la consonante líquida es oclusiva: J 100, Ma 401, 300 y 406; H 200 y 201, en el caso de albahaca; J 100 y Gr 511, para polvareda.

2.6.2. En los Textos. Hemos encontrado 34 casos de $/ \mathrm{lb} /$ en interior de palabra y 40 en margen de palabra. Los resultados son los siguientes:

1. En interior de palabra, [-1] se realiza como [-r] en un 56\%; se mantiene como [-1] en el $17,5 \%$, y es pronunciada como [ $\left[\begin{array}{l}1 \\ 1\end{array}\right]$ en el $26,5 \%$.

2. En frontera léxica, se mantiene como [-1] en el $52,5 \%$ de los casos; se realiza como [-r] en el $45 \%$, y como [l] $\left.\begin{array}{l}1 \\ 1\end{array}\right]$ sólo en un $2,5 \%$.

\subsection{Secuencia heterosilábica [-rt-]}

2.7.1. En el ALEA. Número de mapas: 3: ensartar (mapa n. ${ }^{\circ} 800$ ), cortafrío (mapa n. ${ }^{\circ}$ 977), despertar (mapa n..$^{\circ}$ 702). El número de casos es de 578.

\begin{tabular}{lcc}
\hline & $-\mathrm{rt}-$ & - lt- \\
\hline $\mathrm{Al}$ & 97,5 & 2,5 \\
$\mathrm{Gr}$ & 100 & 1,4 \\
$\mathrm{~J}$ & 98,6 & \\
$\mathrm{Co}$ & 100 & 1,5 \\
$\mathrm{Ma}$ & 98,5 & \\
$\mathrm{Se}$ & 100 & \\
$\mathrm{Ca}$ & 100 & 0,7 \\
$\mathrm{Hu}$ & 100 & \\
And. & 99,3 & \\
\hline
\end{tabular}

1. El porcentaje de mantenimiento de la vibrante es muy alto: el $99,3 \%$, siendo las provincias de $\mathrm{Gr}, \mathrm{Se}, \mathrm{Ca}$ y $\mathrm{Hu}$ las que siempre la han conservado.

2. La realización como [-1] tiene una frecuencia muy baja: el $0,7 \%$.

2.7.2. En los Textos. En interior de palabra, 128 casos; en final de palabra, 15. Los resultados son los siguientes:

1. En interior de palabra, se mantiene la vibrante en el $98,5 \%$ de los casos; en el resto, se reparte por igual entre su pérdida y su realización como [ [1 1 ]

2. En final de palabra: se pierde en el $66,6 \%$; se mantiene en el $26,6 \%$, y se realiza como $\left[\begin{array}{l}1 \\ I\end{array}\right]$ en el resto. 


\subsection{Secuencia heterosilábica [-rd-]}

2.8.1. En el ALEA. Número de mapas: 3: gordo (mapa n. ${ }^{\circ}$ 1299), morder (mapa n. ${ }^{\circ} 1222$ ), escardar (mapa $n^{\circ}{ }^{\circ} 32$ ). El número de casos estudiados es de 646 .

\begin{tabular}{lccc}
\hline & -rd- & -ld- & $-\frac{1}{1} \mathrm{~d}-$ \\
\hline $\mathrm{Al}$ & 100 & & \\
$\mathrm{Gr}$ & 97,6 & 0,7 & 1,6 \\
$\mathrm{~J}$ & 100 & & \\
$\mathrm{Co}$ & 100 & 11,2 & \\
$\mathrm{Ma}$ & 88,7 & & \\
$\mathrm{Se}$ & 100 & & \\
$\mathrm{Ca}$ & 100 & 1,5 & 0,3 \\
$\mathrm{Hu}$ & 100 & & \\
$\mathrm{And}$ & 98,1 & & \\
\hline
\end{tabular}

1. Como en el caso anterior, el porcentaje de mantenimiento de la vibrante es muy alto: el $98,1 \%$; siempre aparece esta líquida en las provincias de $\mathrm{Al}, \mathrm{J}, \mathrm{Co}, \mathrm{Se}, \mathrm{Ca}$ y $\mathrm{Hu}$.

2. El resto de las soluciones, a mucha distancia de la anteriormente mencionada, se reparten entre [ld], con un $1,5 \%$, y $\left[{ }_{1}^{1} d\right]$, con un $0,3 \%$.

3. Hay también realizaciones oclusivas de /d/ en la secuencia $[\mathrm{rd}]$ en $\mathrm{J}$ 500, Al 405, Gr 306 para escardar; Ma 600 para morder; Ca 302 y 101 para gordo.

2.8.2. En los Textos. Número de casos en interior de palabra: 85; en final de palabra: 31 . Los resultados son los siguientes:

1. En interior de palabra, se mantiene la vibrante en el $99 \%$ de los casos, y se realiza como [1] en el resto.

2. En posición final de palabra, se mantiene [-r] en el $32 \%$; se pierde en un $58 \%$, y se realiza como [1] en el resto de los casos.

3. Hemos encontrado la realización oclusiva de [d] en Se 501: [ordenó].

\subsection{Secuencia heterosilábica [-lt-]}

2.9.1. En el ALEA. Número de mapas: 3: saltar (mapa n. ${ }^{\circ} 1422$ ), multa (mapa $\mathrm{n}^{\circ}{ }^{1590)}$ ), el tren (mapa $\left.{ }^{\circ}{ }^{\circ} 1610\right)$. El número de casos es de 628 . 


\begin{tabular}{llllll}
\hline & - lt- & -rt- & $-\frac{1}{\mathrm{I}} \mathrm{t}-$ & -tt- & -ht- \\
\hline $\mathrm{Al}$ & 25,7 & 60 & 11,4 & 2,8 & \\
$\mathrm{Gr}$ & 12,6 & 67 & 19,3 & & 0,8 \\
$\mathrm{~J}$ & 31 & 57 & 11,6 & & \\
$\mathrm{Co}$ & 13,7 & 82 & 4,1 & & \\
$\mathrm{Ma}$ & 14,5 & 75 & 6,5 & 4 & 1,2 \\
$\mathrm{Se}$ & 11,6 & 81,4 & 5,8 & & \\
$\mathrm{Ca}$ & 12,5 & 82 & 5,5 & & \\
$\mathrm{Hu}$ & 32 & 58 & 9,8 & & 0,3 \\
And. & 18,8 & 70 & 10 & 0,8 & \\
\hline
\end{tabular}

1. La realización más frecuente de la lateral es [-r], con el $70 \%$.

2. Siguen a la anteriormente mencionada el mantenimiento de [-1], con un $18,8 \%$, y la articulación como $\left[\begin{array}{l}1 \\ 1\end{array}\right]$, con un $10 \%$.

3. Muy bajas son las frecuencias de las restantes realizaciones: de [tt], el $0,8 \%$ y de $[\mathrm{h}]$ el $0,3 \%$.

2.9.2. En los Textos. El número total de casos es de 89 , repartidos de la siguiente manera: 42 en interior de palabra, y 47 en frontera léxica. Los resultados son los siguientes:

1. En interior de palabra, [-1] se realiza como [-r] en un $74 \%$, y como lateral, en el $26 \%$ restante.

2. En final de palabra, se realiza como [-r] en un $57,5 \%$ de casos; como [-1], en un $38 \%$, y como $\left[\begin{array}{l}1 \\ 1\end{array}\right]$ en $4,5 \%$.

\subsection{Secuencia heterosilábica [-ld-]}

2.10.1. En el ALEA. Número de mapas: 4: aldea (mapa n..$^{\circ} 870$ ), espal$d a$ (mapa $\mathrm{n}^{\circ}$ 1250), aldaba (mapa $\mathrm{n}^{\circ}$ 663), tarabilla (con respuesta mayoritaria «aldaba») (mapa n. ${ }^{\circ} 666$ ). El número de casos es de 628.

\begin{tabular}{lrllll}
\hline & -ld- & -rd- & -1 d- & -nd- & -Ød- \\
\hline $\mathrm{Al}$ & 32,3 & 60 & 7,6 & & \\
$\mathrm{Gr}$ & 7,1 & 83,6 & 9,1 & & \\
$\mathrm{~J}$ & 43,5 & 52,9 & 2,3 & 1,1 & \\
$\mathrm{Co}$ & 3,7 & 90,1 & 4,9 & & 1,2 \\
$\mathrm{Ma}$ & 19,7 & 77,4 & 2,9 & & \\
$\mathrm{Se}$ & 4,9 & 86,2 & 8,8 & & \\
$\mathrm{Ca}$ & 4,1 & 91,6 & 4,1 & & \\
$\mathrm{Hu}$ & 8,9 & 85,8 & 5,1 & & 0,1 \\
$\mathrm{And}$ & 15,2 & 78,5 & 5,8 & 0,1 & 0 \\
\hline
\end{tabular}


1. Como en el caso anterior, el porcentaje más alto de realizaciones está constituido por la pronunciación [-r] de la lateral, con un $78,5 \%$.

2. Siguen, a mucha distancia, el mantenimiento de [1], con un $15,2 \%$, y su realización $\left[\begin{array}{l}1 \\ 1\end{array}\right]$, con un $5,8 \%$.

3. Con un $0,1 \%$ aparecen los casos de pérdida y de realización como nasal.

2.10.2. En los Textos. En posición interior de palabra, 12 casos; en posición final, 36. Los resultados son los siguientes:

1. En interior de palabra, [-1] es [-r] en el $50 \%$ de los casos; se mantiene como lateral en el $42 \%$, y como $\left[\begin{array}{l}1 \\ I\end{array}\right]$, en el $8 \%$.

2. En final de palabra: se mantiene como [-1] en un $72 \%$ de los casos; se pronuncia como [-r] en un $11 \%$; se pierde en el $14 \%$; el resto se reparte entre soluciones minoritarias.

\subsection{Secuencia heterosilábica [-rk-]}

2.11.1. En el ALEA. Número de mapas: 3: marco (mapa n. ${ }^{\circ}$ 665), cerco de la luna (mapa n. ${ }^{\circ} 844$ ), arco iris (mapa n. ${ }^{\circ} 860$ ). El número de casos estudiados es de 681 .

\begin{tabular}{lcccccc}
\hline & -rk- & -lk- & $-1 \mathrm{k}$ k- & -ik- & -hk- & -lk- \\
\hline $\mathrm{Al}$ & 99 & 1 & & & & \\
$\mathrm{Gr}$ & 97,7 & 0,7 & & 0,7 & 0,7 & \\
$\mathrm{~J}$ & 100 & & & & & \\
$\mathrm{Co}$ & 100 & & 1,2 & 2,4 & & 1,2 \\
$\mathrm{Ma}$ & 90 & 5 & & & & \\
$\mathrm{Se}$ & 100 & & & & & \\
$\mathrm{Ca}$ & 98 & 2 & & 0,4 & 0,1 & 0,1 \\
$\mathrm{Hu}$ & 98,5 & 1,5 & & & \\
And. & 98 & 1,1 & 0,1 & 0,4 & \\
\hline
\end{tabular}

1. Predomina el mantenimiento de la vibrante, en el $98 \%$ de los casos.

2. Siguen las realizaciones minoritarias de [-1], con un $1,1 \%$, [i] con $0,4 \%,\left[\begin{array}{l}1 \\ \mathrm{I}\end{array}\right.$, con $0,1 \%,[\mathrm{~h}]$ con $0,1 \%$ y [l] también con $0,1 \%$.

2.11.2. En los Textos, 107 casos repartidos del siguiente modo: 72 en interior de palabra; 35 en final de palabra. Los resultados son los siguientes:

1. En interior de palabra, predomina el mantenimiento de la vibrante en un $87,5 \%$. Se pierde en un $4,2 \%$, y con un porcentaje similar, se aspira. Con un $2,7 \%$, se realiza como [-k], y con un $1,3 \%$, como [-1]. 
2. En final de palabra, se pierde en un $46 \%$; se realiza como [-r] en un $31,5 \%$. Es $[-1]$ en un $8,5 \%$, y $\left.{ }_{1}^{1}\right]$, también con un $8,5 \%$. El resto se reparte en soluciones minoritarias.

\subsection{Secuencia heterosilábica [-rg-]}

2.12.1. En el ALEA. Número de mapas: 3: garganta (mapa n. ${ }^{\circ}$ 1226), alpargata (mapa n. ${ }^{\circ}$ 1407), juerga (mapa n. $^{\circ} 1585$ ). El número de casos es de 681.

\begin{tabular}{lcccccc}
\hline & $-\mathrm{rg}-$ & $-\mathrm{lg}-$ & $-1 \mathrm{~g}-$ & $-\mathrm{gg}-$ & $-\mathrm{ig}-$ & $-\varnothing \mathrm{g}-$ \\
\hline $\mathrm{Al}$ & 98 & 2 & & & & \\
$\mathrm{Gr}$ & 98 & 2 & & & & \\
$\mathrm{~J}$ & 89 & 5,5 & 5,5 & & & \\
$\mathrm{Co}$ & 98,5 & 1,5 & & & & \\
$\mathrm{Ma}$ & 87 & 7,5 & 1,2 & 2,5 & 1,2 & \\
$\mathrm{Se}$ & 99 & & 1 & & & \\
$\mathrm{Ca}$ & 100 & & & & & 3 \\
$\mathrm{Hu}$ & 97 & & & 0,2 & 0,1 & 0,2 \\
$\mathrm{And}$ & 95,8 & 2,5 & 1 & & & \\
\hline
\end{tabular}

1. Predomina el mantenimiento de la vibrante, con un $95,8 \%$.

2. La realización como [-1] es del $2,5 \%$, y como $\left[\begin{array}{l}1 \\ 1\end{array}\right]$, del $1 \%$.

3. Porcentajes mínimos suponen las realizaciones como [gg] $(0,2 \%)$, como [ig] $(0,1 \%)$, o su desaparición, en un $0,2 \%$.

4. [g] es oclusiva después de las líquidas en $\mathrm{Al} 400,401,403,405$, 303, 506, 508; Gr 503, 508, 515; Ma 300, 406; Se 310, 603; Ca 302; H 303, para garganta. Ca 300 para juerga.

2.12.2. En los Textos. El número de casos de esta secuencia [rg] es muy pequeño: 33 en posición interior de palabra, y 3 en final.

1. En posición interior, se mantiene la vibrante en un $97 \%$; el $3 \%$ restante es para la realización como [-1].

2. En posición final de palabra, se pierde siempre.

\subsection{Secuencia heterosilábica [-lk-].}

2.13.1. En el ALEA. Número de mapas: 4: alcohol (mapa n. ${ }^{\circ}$ 1617), alcaparra (mapa n. 300 ), alcachofa (mapa n. ${ }^{\circ} 323$ ) y el cular (mapa n. $^{\circ}$ 1618). Número de casos: 784. 


\begin{tabular}{lllllllll}
\hline & $-1 \mathrm{k}-$ & $-\mathrm{rk}-$ & $-{ }_{1}^{1} \mathrm{k}-$ & $-\mathrm{kk}-$ & $-{ }_{I}^{\mathrm{h} k-}$ & - hk- & $-\varnothing \mathrm{k}-$ & - ik- \\
\hline $\mathrm{Al}$ & 30 & 58 & 9 & 2 & 1 & & & \\
$\mathrm{Gr}$ & 18,1 & 53 & 20 & 2 & 1 & 5,5 & & \\
$\mathrm{~J}$ & 29,7 & 54,7 & 15 & & 0,7 & & 0,7 & \\
$\mathrm{Co}$ & 11 & 71 & 13,5 & & & 1 & 3,3 & \\
$\mathrm{Ma}$ & 24 & 66,6 & 8 & & & & & 1 \\
$\mathrm{Se}$ & 12 & 78 & 8 & 2 & & & & \\
$\mathrm{Ca}$ & 8,7 & 84 & 1,7 & & 3,5 & 1,7 & & \\
$\mathrm{Hu}$ & 23,6 & 71 & 4 & 1,4 & & & & \\
And. & 20,1 & 64,6 & 11,5 & 1 & 0,7 & 1,4 & 0,5 & 0,1 \\
\hline
\end{tabular}

1. Predomina la realización de [-1], como [-r], con un $64,6 \%$

2. Sigue el mantenimiento de [-1], con un $20,1 \%$, y de $\left[\begin{array}{l}1 \\ 1\end{array}\right]$, con un $11,5 \%$.

3. Con porcentajes muy pequeños, están las realizaciones [h], con $1,4 \%$, [kk], con un $1 \%$, [ $\left.{ }_{I}^{\mathrm{h}}\right]$, con el $0,7 \%$, su pérdida, en un $0,5 \%$, y la realización como semivocal, [i] , con un $0,1 \%$.

2.13.2. En los Textos, el número de casos es muy desigual: 10 en interior de palabra y 90 en el margen léxico. Los resultados son los siguientes:

1. En posición interior de palabra, predomina la realización de [-1] como [-r]: un $70 \%$; sigue el mantenimiento de la lateral con el $20 \%$, y, por último, la realización $\left[\begin{array}{l}1 \\ 1\end{array}\right]$, con el $10 \%$.

2. En final de palabra, predomina el mantenimiento de la lateral, con un $57 \%$. El $24,5 \%$ viene dado por la realización [-r]. Se pierde en un $11 \%$; se pronuncia como [lr] en un 5,5\%, y el resto se articula como [-k].

\subsection{Secuencia heterosilábica [-lg-].}

2.14.1. En el ALEA. Sólo 1 mapa: acelga (mapa n. ${ }^{\circ} 328$ ). El número de casos estudiados es de 204.

\begin{tabular}{lccc}
\hline & $-\lg -$ & - rg- & $-\frac{1}{1} \mathrm{~g}-$ \\
\hline $\mathrm{Al}$ & 13,7 & 72,4 & 13,7 \\
$\mathrm{Gr}$ & 13,1 & 76,3 & 10,5 \\
$\mathrm{~J}$ & 10,3 & 75,8 & 13,7 \\
$\mathrm{Co}$ & 8 & 88 & 4 \\
$\mathrm{Ma}$ & 20 & 72 & 8 \\
$\mathrm{Se}$ & & 100 & \\
$\mathrm{Ca}$ & 17,3 & 94,1 & 5,8 \\
$\mathrm{Hu}$ & 11,2 & 78,2 & 4,3 \\
$\mathrm{And}$ & & 80,3 & 8,3 \\
\hline
\end{tabular}


1. Predomina la realización [-r], con un $80,3 \%$.

2. Siguen las realizaciones de [1], con un $11,2 \%$, y de $\left[\begin{array}{l}1 \\ I\end{array}\right]$, con un $8,3 \%$.

3. Como en casos anteriores, [g] se ha pronunciado como oclusiva, después de una consonante líquida en los siguientes puntos: Se 102, 401; Hu 504; Gr 308; Co 300, 401, 606; Ma 100, 101; Al 203.

2.14.2. En los Textos, sólo se han presentado 44 casos: 31 en interior de palabra y 13 en final de palabra. Los resultados son los siguientes:

1. En posición interior de palabra, la lateral se realiza como [-r] en el $71 \%$ de los casos; se mantiene como [-1] en el $26 \%$, y como [ $\left[\begin{array}{l}1 \\ 1\end{array}\right]$, en el resto.

2. En el margen léxico, se mantiene como [-1] en el $61,5 \%$, y se articula como [-r] en el $38,5 \%$.

\subsection{Secuencia heterosilábica $[-l f-]^{10}$}

2.15.1. En el ALEA. Sólo 1 mapa: alfiler (mapa n. ${ }^{\circ} 1746$ ). El número de casos estudiados es de 228.

\begin{tabular}{llllll}
\hline & -lf- & -rf- & $-\frac{1}{x}$ f- & -ff- & -Øf- \\
\hline $\mathrm{Al}$ & 34,4 & 51,7 & 13,7 & & \\
$\mathrm{Gr}$ & 32,6 & 50 & 17,3 & & \\
$\mathrm{~J}$ & 32,2 & 54,8 & 9,6 & 3,2 & 4 \\
$\mathrm{Co}$ & 24 & 68 & 4 & & \\
$\mathrm{Ma}$ & 29,6 & 59,2 & 11,1 & & \\
$\mathrm{Se}$ & 10 & 80 & 10 & & \\
$\mathrm{Ca}$ & 6,2 & 93,7 & & & \\
$\mathrm{Hu}$ & 45,8 & 54,1 & & & \\
And. & 28 & 61,4 & 9,6 & 0,4 & 0,4 \\
\hline
\end{tabular}

1. La realización predominante de la lateral es [-r], con un $61,4 \%$.

2. Sigue el mantenimiento de [-1], con el $28 \%$, y, después, de $\left[\begin{array}{l}1 \\ I\end{array}\right]$, con el $9,6 \%$.

3. A mucha distancia, con un $0,4 \%$ cada una, [ff] y su desaparición.

2.15.2. En los Textos, el número de ocurrencias es escaso: 6 en interior de palabra, y 21 en final. Los resultados muestran que:

1. En interior de palabra, la lateral se realiza como [1] en el $83 \%$ de los casos, y se pierde en el resto.

10 A veces, como nos ha ocurrido en el caso de la secuencia [rf], o no hemos encontrado mapas que la contuviesen, o los mapas eran sintéticos. 
2. En posición final de palabra, se realiza como [-r] en el $66,6 \%$; como [-1], en el $19 \%$; se pierde, en un $9,5 \%$, y se realiza como [Ф] en el resto ${ }^{11}$.

\subsection{Secuencia heterosilábica $[-r \theta-]^{12}$}

2.16.1. En el ALEA. Número de mapas: 4: barzón (mapa n. ${ }^{\circ}$ 124), murciélago (mapa n. ${ }^{\circ} 425$ ), zarzas (mapa n..$^{\circ} 1642$ ), porción (mapa $n .^{\circ} 1727$ ). Número de casos: 848 .

\begin{tabular}{lcccccc}
\hline & $-\mathrm{r} \theta-$ & $-\mathrm{rs}-$ & $-1 \mathrm{~s}-$ & $-\varnothing \theta-$ & $-\varnothing \mathrm{s}-$ & $-1 \theta-$ \\
\hline $\mathrm{Al}$ & 100 & & & & & \\
$\mathrm{Gr}$ & 99 & & 1 & & & \\
$\mathrm{~J}$ & 88 & 10 & 0,9 & 0,9 & & \\
$\mathrm{Co}$ & 54 & 45 & & & 1 & \\
$\mathrm{Ma}$ & 75,5 & 15,5 & 1 & 5 & 1 & 2 \\
$\mathrm{Se}$ & 79,5 & 17 & & 0,8 & 2,5 & \\
$\mathrm{Ca}$ & 94 & 3 & & 3 & & \\
$\mathrm{Hu}$ & 88 & 11 & & 1 & & \\
And. & 85,5 & 12 & 0,5 & 1,2 & 0,6 & 0,2 \\
\hline
\end{tabular}

Las secuencias heterosilábicas de las líquidas implosivas seguidas de [ $\theta]$ o de [s] dan resultados complejos, por las mencionadas soluciones andaluzas de distinción, de ceceo y de seseo.

En el caso que nos ocupa, los resultados son los siguientes:

1. Mantenimiento de [-r], con un $97,55 \%$ de casos, desglosado en: [ $\mathrm{r} \theta]$, con un $85,5 \%$, y [rs], con un $12 \%$.

2. La realización como [-1] muestra una frecuencia muy pequeña: el $0,7 \%$, que se reparte entre [1s], con el $0,5 \%$ y [10], con el $0,2 \%$.

3. Los casos de pérdida son también pocos: un $1,8 \%$.

4. Almería mantiene siempre [ $\mathrm{r} \theta]$.

11 No hemos podido analizar la secuencia [rf] en el $A L E A$. En los Textos, hemos encontrado sólo 4 casos de [rf] en margen de palabra; de ellos, el 50\% mantenía [-r] y el otro $50 \%$ la perdía.

${ }^{12}$ En Andalucía, como es sabido, existen tres áreas en lo que se refiere al comportamiento de los fonemas /s/y / / /: a) una área de distinción entre ambos fonemas que se da en la mayor parte de las provincias de Jaén y Almería; también se da en el norte de Huelva, de Córdoba y este de Granada; b) el ceceo se extiende por casi todo el sur de Andalucía, desde la frontera de Portugal hasta Almería. Comprende toda la provincia de Cádiz, gran parte de Sevilla, gran parte del sur de Huelva, sur de Málaga, gran parte de Granada y sur de Almería; c) el seseo se produce en zonas al noreste de Huelva, norte de Sevilla, de Málaga y gran parte del sur de Córdoba. (Vid. Alonso Zamora Vicente, Dialectología Española, Madrid,

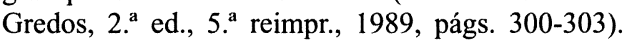


2.16.2. En los Textos, hemos reunido las secuencias normativas [ $\mathrm{r} \theta]$ y [rs], debido al no muy elevado número de casos y a las diferentes realizaciones de las fricativas explosivas. Han aparecido 78 casos, repartidos del siguiente modo: 62 en interior de palabra y 16 en final. Los resultados son:

1. En interior de palabra, [-r] se mantiene en el $77,5 \%$ de los casos: en la secuencia [r $\theta$ ], con un $56,5 \%$, y en la secuencia [rs], con un $21 \%$. Se pierde en el $17,7 \%$, tanto ante $[\theta]$, como ante [s]; aparece realizada como [1] en el 4,8\%.

2. En posición final de palabra, se mantiene [-r] ante [s] en el $37,5 \%$; se pierde, tanto ante $[\theta]$, como ante [s], en el $43,7 \%$. Se pronuncia como [1] en $12,5 \%$; el resto se reparte entre soluciones minoritarias.

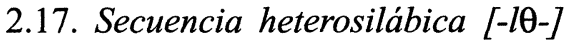

2.17.1. En el ALEA. Número de mapas: 4: descalzo (mapa n. ${ }^{\circ}$ 1589), calzar la reja (mapa n..$^{\circ} 148$ ), calzoncillo (mapa $\mathrm{n}^{\circ}$ 1393), calcetín (mapa n. $^{\circ}$ 1403). El número de casos estudiados es de 875 .

\begin{tabular}{lcclccccccc}
\hline & $-1 \theta-$ & $-1 s-$ & $-r \theta-$ & $-r s-$ & $-\frac{1}{\perp} \theta-$ & $-\frac{1}{\mathrm{I}} \mathrm{s}-$ & $-\varnothing \theta-$ & $-\varnothing \mathrm{s}-$ & $-\mathrm{n} \theta-$ & $-\mathrm{h} \theta-$ \\
\hline $\mathrm{Al}$ & 13 & & 82 & & 5 & & & & & \\
$\mathrm{Gr}$ & 6,7 & 1,2 & 84 & & 7,3 & & & & 0,6 & \\
$\mathrm{~J}$ & 15 & & 78,5 & 1,7 & 3,5 & & 1 & & & \\
$\mathrm{Co}$ & 3,8 & 2 & 57 & 29 & 3 & 3,8 & & 1 & & \\
$\mathrm{Ma}$ & 6,5 & 3,7 & 74,7 & 9,3 & 4,6 & 1 & & & & \\
$\mathrm{Se}$ & 3,3 & 2,5 & 78,3 & 11,6 & 0,8 & 1,6 & & 1,6 & & \\
$\mathrm{Ca}$ & 4,3 & 1,5 & 84 & 4,3 & 2,8 & & & & 1,5 & 1,5 \\
$\mathrm{Hu}$ & 9,5 & 1,2 & 76 & 7 & 3,5 & 1,2 & & & 1,2 & \\
And. & 7,8 & 1,5 & 77 & 7,4 & 4 & 1 & 0,1 & 0,3 & 0,3 & 0,1 \\
\hline
\end{tabular}

1. El porcentaje más alto es la realización como vibrante, con el $84,4 \%$, desglosado del siguiente modo: [r $\mathrm{r}]$, con un $77 \%$, y [rs] con un $7,4 \%$.

2. Sigue a mucha distancia la realización como $[-1]$, con un porcentaje del $9,3 \%$ repartido entre [10], con el $7,8 \%$ y [ls], con el $1,5 \%$.

3. A continuación, $\left[\begin{array}{l}1 \\ I\end{array}\right]$, con un $5 \%:\left[{ }_{I}^{1} \theta\right]$ con el $4 \%$ y $\left[{ }_{I}^{1} \mathrm{~s}\right]$ con el $1 \%$.

4. El resto de las soluciones tienen frecuencias bajísimas.

2.17.2. En los Textos, por las mismas razones que exponíamos en el anterior $\S 2.16 .2$., hemos reunido las secuencias normativas [10] y [1s]. Sólo han aparecido 53 casos, repartidos muy desigualmente: 5 en interior de palabra, y 48 en final. Los resultados son los siguientes: 
1. En interior de palabra, hay 2 casos de realización de [ls] y 3 de [r $\theta]$.

2. En final de palabra, se mantiene la lateral en un $62,5 \%$; se realiza como [-r] en un $27,5 \%$, tanto ante [s], como ante [ $\theta]$; se pierde en un $8 \%$; el resto, se manifiesta en soluciones minoritarias.

\subsection{Secuencia heterosilábica [-rs-]}

2.18.1. En el ALEA. Cuatro mapas: oxidarse (mapa n. ${ }^{\circ}$ 978), secarse (mapa $n . .^{\circ} 853$ ), desperezarse (mapa $n .{ }^{\circ}$ 1242), rascarse (mapa $n .^{\circ} 1281$ ). Número de casos: 772.

\begin{tabular}{lcccccccc}
\hline & $-\varnothing \mathrm{s}-$ & $-1 \mathrm{~s}-$ & $-\mathrm{rs}-$ & $-\varnothing \theta-$ & $-\mathrm{r} \theta-$ & $-\frac{1}{1} \mathrm{~s}-$ & $-1 \theta-$ & $-\frac{1}{1} \theta-$ \\
\hline $\mathrm{Al}$ & 44 & 20 & 24 & 10 & 1 & 1 & & \\
$\mathrm{Gr}$ & 39 & 4,5 & 9,5 & 42,5 & 4,5 & & & \\
$\mathrm{~J}$ & 80 & 3,6 & 10 & 2,7 & 1,8 & 1,8 & & \\
$\mathrm{Co}$ & 63,5 & 2,2 & 19,3 & 11,4 & 1,1 & 2,2 & & \\
$\mathrm{Ma}$ & 6,4 & 6,4 & 13,8 & 37 & 34 & & 2 & \\
$\mathrm{Se}$ & 20 & 2 & 13,8 & 16 & 45,5 & 1 & & 1 \\
$\mathrm{Ca}$ & 2 & & 4 & 12 & 82 & & & \\
$\mathrm{Hu}$ & 47 & 5 & 13 & 22 & 13 & & & \\
And. & 40,5 & 6 & 13,6 & 21 & 17,7 & 0,7 & 0,2 & 0,1 \\
\hline
\end{tabular}

1. Como era de esperar en esta secuencia, predomina la pérdida de $[-\mathrm{r}]^{13}$, con una frecuencia del 61,5\%; lógicamente, es superior cuando va seguida de [s], con el $40,5 \%$, que cuando es [ $\theta$ ] el sonido que sigue a la vibrante, con el $21 \%$.

2. En segundo lugar, se produce el mantenimiento de [-r], con un $31,3 \%$, repartido muy por igual entre las dos secuencias: [rs], con el $13,6 \%$ $\mathrm{y}$ [r $\theta$ ], con el $17,7 \%$.

3. A bastante distancia, se da la realización [-1], con un $6,02 \%$ : [1s], $6 \%$ y $[1 \theta], 0,2 \%$.

4. El resto de las realizaciones tienen frecuencias muy bajas.

\subsection{Secuencia heterosilábica $[-r x-]^{14}$}

2.19.1. En el ALEA. Dos mapas: alfarje (mapa n. ${ }^{\circ} 135$ ), y los significantes correspondientes al 'primer aceite' (mapa n. $\left.{ }^{\circ} 238\right)^{15}$. El número de casos estudiados es de: 219 .

\footnotetext{
${ }^{13}$ En todos los casos, el mantenimiento de [-r] en esta secuencia da unos porcentajes pequeños. La pérdida de la vibrante es una tendencia manifestada ya en el mismo latín. Vid. R. Menéndez Pidal, Manual de Gramática Histórica Española, Madrid, 1958, pág. 136.

14 Tanto [-rx-], como [-rh-] o variantes.

15 Donde predomina como respuesta «aceite virgen».
} 


\begin{tabular}{lllllr}
\hline & -rx- & $-{ }_{1}^{1} \mathrm{x}$ & -nx- & - Øh- & vn h \\
\hline $\mathrm{Al}$ & 80 & 6,6 & 13,3 & & \\
$\mathrm{Gr}$ & 47 & & 11,7 & 35,3 & 6 \\
$\mathrm{~J}$ & 57 & & 43 & & \\
$\mathrm{Co}$ & 22 & & 28 & 30,5 & 19,5 \\
$\mathrm{Ma}$ & 46,6 & & 23,3 & 16,6 & 13,3 \\
$\mathrm{Se}$ & 13,8 & & 13,8 & 52,7 & 19,4 \\
$\mathrm{Ca}$ & 28,5 & & & 42,8 & 28,5 \\
$\mathrm{Hu}$ & 29,5 & & 17,6 & 47 & 5,8 \\
And. & 39,7 & 0,5 & 22,8 & 26,5 & 10,5 \\
\hline
\end{tabular}

1. [-r] se mantiene en el $39,7 \%$ de los casos, y se pierde en el $26,5 \%$.

2. Se realiza como [-n] en el $22,8 \%$ de los casos.

3. Se realiza como [h], nasalizando la vocal núcleo silábico, en un $10,5 \%$

4. Y, por último, aparece como $\left[\begin{array}{l}1 \\ 1\end{array}\right]$ en un $0,5 \%$.

2.19.2. En los Textos, sólo hemos encontrado 7 casos de esta secuencia: 6 en posición interior de palabra, en los que siempre se conserva la [-r], y 1 en posición final de palabra, donde se pierde la vibrante ${ }^{16}$.

\subsection{Secuencia heterosilábica [-lî-].}

2.20.1. En el ALEA, sólo un mapa: colchón (mapa n. ${ }^{\circ} 1582$ ). Número de casos: 231 .

\begin{tabular}{lccc}
\hline & $-r \hat{c}-$ & $-1 \hat{c}-$ & $-\frac{1}{d} \hat{\mathrm{c}}-$ \\
\hline $\mathrm{Al}$ & 74,1 & 12,9 & 12,9 \\
$\mathrm{Gr}$ & 69,5 & 17,3 & 13 \\
$\mathrm{~J}$ & 52,9 & 32,3 & 14,7 \\
$\mathrm{Co}$ & 100 & & \\
$\mathrm{Ma}$ & 80,7 & 7,6 & 11,5 \\
$\mathrm{Se}$ & 93,3 & 3,3 & 3,3 \\
$\mathrm{Ca}$ & 100 & & \\
$\mathrm{Hu}$ & 90,9 & 9 & 8,2 \\
And. & 79,6 & 12,1 & \\
\hline
\end{tabular}

${ }^{16}$ En los Textos, hemos encontrado también la secuencia [-1/x-], sólo en posición final de palabra, en 8 casos. En 4, se ha perdido la lateral; en 3, se ha conservado, y en 1 se ha realizado como [-r]. 
1. La realización de la lateral como [-r] reúne el $79,6 \%$ de los casos. En las provincias de $\mathrm{Co}$ y $\mathrm{Ca}$, asciende al $100 \%$, y en $\mathrm{Se}$ y $\mathrm{Hu}$, al 93,3 y $90,9 \%$, respectivamente.

2. Siguen el mantenimiento de [-1], con un $12,1 \%$ y la realización $\left[\begin{array}{l}1 \\ 1\end{array}\right]$, con un $8,2 \%$.

2.20.2. En los Textos, sólo hemos encontrado 1 caso de esta secuencia en posición interior de palabra con mantenimiento de $[-1]{ }^{17}$.

\subsection{Secuencia heterosilábica [-rm-].}

2.21.1. En el ALEA. Número de mapas: 2: hormiga (mapa n. ${ }^{\circ} 391$ ), Carmen (mapa n. ${ }^{\circ}$ 1579). El número de casos estudiados es de 448.

\begin{tabular}{lllcccc}
\hline & $-\mathrm{rm}-$ & $-\mathrm{lm}-$ & $-\frac{1}{\mathrm{I}} \mathrm{m}-$ & $-\mathrm{mm}-$ & $-\tilde{\mathrm{hm}}-$ & $-\mathrm{x} \tilde{\mathrm{hm}}-$ \\
\hline $\mathrm{Al}$ & 80 & 10 & 6,5 & 1,6 & 1,6 & \\
$\mathrm{Gr}$ & 66,6 & 7,7 & 19,2 & 1,3 & 5 & \\
$\mathrm{~J}$ & 87 & 6,5 & 5 & 1,5 & & \\
$\mathrm{Co}$ & 71 & 11 & 12,5 & 1,5 & 1,5 & 1,5 \\
$\mathrm{Ma}$ & 70 & 15 & 9,5 & 1,8 & 1,8 & 1,8 \\
$\mathrm{Se}$ & 97 & & & & 1,5 & 1,5 \\
$\mathrm{Ca}$ & 97 & & & & 3 & \\
$\mathrm{Hu}$ & 89,2 & 6,4 & & 2 & 2 & \\
And. & 80,4 & 7,4 & 7,6 & 1,3 & 2,2 & 0,6 \\
\hline
\end{tabular}

1. Se mantiene [-r] en un $80,4 \%$ de los casos.

2. Se realiza como $[-1]$ en un $7,4 \%$, y como $\left[\begin{array}{l}1 \\ 1\end{array}\right]$ en un $7,6 \%$.

3. Sigue la realización como aspirada nasal $[\widetilde{\mathrm{h}}]$, con un $2,2 \%$, seguida de $[\mathrm{mm}]$ o de $[\mathrm{m}]$, en orden decreciente de frecuencia.

4. La realización de la secuencia $[\mathrm{rm}]$ como $[\mathrm{mm}]$ tiene una frecuencia del $1,3 \%$, etc.

2.21.2. En los Textos, han aparecido 66 casos: 54 en interior de palabra, y 12 en final. Los resultados son los siguientes:

1. En posición interior de palabra, se ha mantenido la vibrante en el $98 \%$ de los casos, realizándose en el resto como [-1].

2. En posición final de palabra, se ha mantenido la vibrante en el $50 \%$ de los casos, y se ha perdido en el otro $50 \%$.

${ }^{17}$ En los Textos, hemos encontrado 16 casos de la secuencia [r $\left.\mathrm{c}\right]: 15$ en interior de palabra, y 1 en final: en todos se conserva la vibrante. 


\subsection{Secuencia heterosilábica [-lm-].}

2.22.1. En el ALEA: 3 mapas: almohada (mapa n. ${ }^{\circ}$ 692), pulmón (mapa n. ${ }^{\circ}$ 1334), salmuera (mapa n. $^{\circ}$ 572). El número de casos estudiados es de 595.

\begin{tabular}{lllrrrrrr}
\hline & $-\mathrm{lm}-$ & $-\mathrm{rm}$ & $-{ }_{I}^{1} \mathrm{~m}-$ & $-\varnothing \mathrm{m}-$ & $-\tilde{\mathrm{hm}}-$ & $-\mathrm{mm}-$ & $-{ }_{I}^{\mathrm{h}} \mathrm{m}-$ & $-{ }_{1}^{\mathrm{h}} \mathrm{m}-$ \\
\hline $\mathrm{Al}$ & 43,7 & 26,5 & 10,9 & 12,5 & 3 & 3,1 & & \\
$\mathrm{Gr}$ & 33 & 28,6 & 21,7 & 9,5 & 6 & & 0,8 & \\
$\mathrm{~J}$ & 43,8 & 16,3 & 13,6 & 20,5 & 2,6 & 1,3 & & 1,3 \\
$\mathrm{Co}$ & 31,9 & 31,8 & 5,5 & 26,3 & 4 & & & \\
$\mathrm{Ma}$ & 36,3 & 28,4 & 12,9 & 14,2 & 5,19 & 2,5 & & \\
$\mathrm{Se}$ & 18,5 & 37 & 16 & 21 & 73 & & & \\
$\mathrm{Ca}$ & 13 & 67,3 & 6,5 & 8,6 & 2,1 & 2,1 & & \\
$\mathrm{Hu}$ & 32,8 & 37,2 & 13,4 & 8,9 & 4,4 & 1,4 & 1,4 & \\
And. & 32,2 & 32,3 & 13,6 & 15,2 & 4,6 & 1,1 & 0,3 & 0,1 \\
\hline
\end{tabular}

1. El mantenimiento de [-1], o su realización como [-r] ${ }^{18}$ tienen frecuencias muy similares: $32,3 \%$, esta última, y $32,2 \%$ la primera.

2. Sigue la pérdida de la lateral, con un $15,2 \%$, y su realización como $\left[\begin{array}{l}1 \\ 1\end{array}\right]$, con un $13,6 \%$.

3. Su pronunciación como aspirada nasal, $[\tilde{\mathrm{h}}]$, tiene un porcentaje muy pequeño, el $4,6 \%$.

4. La realización de $[\mathrm{lm}]$ como $[\mathrm{mm}]$ aparece con un $1,1 \%$.

5. Y por último, con porcentajes muy pequeños las realizaciones de: $\left[\begin{array}{l}\mathrm{h} \\ \mathrm{I}\end{array}\right]$, con el $0,3 \%$, y de $\left[\begin{array}{l}\mathrm{h} \\ 1\end{array}\right]$, con el $0,1 \%$.

2.22.2. En los Textos, hemos encontrado 107 casos de esta secuencia, repartidos del siguiente modo: 31 en posición interior de palabra, y 76 en final. Los resultados son los siguientes:

1. En interior de palabra, la lateral se realiza como [-r] en el $58 \%$ de los casos; como [ [ $\left.\begin{array}{l}1 \\ 1\end{array}\right]$, en el $26 \%$, y como [-1], en el $16 \%$.

2. Cuando es final de palabra, se realiza como [-r] en el $62 \%$ de los casos; como [-1], en el $33 \%$, y con un $2,5 \%$, cada una, como [ $\left.{ }_{1}^{1}\right]$ y su desaparición.

\subsection{Secuencia heterosilábica [-rn-]}

2.23.1. En el ALEA. Número de mapas: 3: carnaval (mapa n. ${ }^{\circ}$ 1476), yerno (mapa $\mathrm{n}^{\circ}$ 1359), sarna (mapa $\mathrm{n}^{\circ}{ }^{\circ} 1280$ ). El número de casos estudiados es de 663 .

\footnotetext{
${ }^{18}$ Esta [r] es fricativa nasal en $1,1 \%$ de los casos.
} 


\begin{tabular}{llcccccc}
\hline & $-\mathrm{rn}-$ & $-\ln -$ & $-\frac{1}{\mathrm{I}} \mathrm{n}-$ & $-{ }_{I}^{\mathrm{h}} \mathrm{n}-$ & $-\mathrm{nn}-$ & $-\tilde{\mathrm{hn}}-$ & $-\frac{\mathrm{h}}{1} \mathrm{n}-$ \\
\hline $\mathrm{Al}$ & 18 & 5,6 & 6,8 & 11,3 & 23,8 & 32,9 & 1,1 \\
$\mathrm{Gr}$ & 20,5 & 3,8 & 0,7 & 14,8 & 16 & 44,2 & \\
$\mathrm{~J}$ & 41,4 & 13,4 & 5,6 & 16,8 & 6,7 & 15,6 & \\
$\mathrm{Co}$ & 31 & 4 & 1,3 & 8,1 & 21,6 & 32,4 & 1,3 \\
$\mathrm{Ma}$ & 12,1 & & 1,3 & 10,8 & 20,2 & 55,3 & \\
$\mathrm{Se}$ & 14,1 & & 1 & 20,8 & 17,5 & 46 & \\
$\mathrm{Ca}$ & 11,1 & & & 20 & 6,6 & 62,1 & \\
$\mathrm{Hu}$ & 28 & 1,4 & 11,2 & 26,7 & & 32,3 & \\
And. & 22,4 & 3,8 & 3,4 & 15,8 & 14,7 & 39 & 0,2 \\
\hline
\end{tabular}

1. La realización más frecuente de [-r] es la de una aspiración nasal, [ $\tilde{\mathrm{h}}$ ]: $39 \%$; en este caso, [n] se articula como [n] o como [nn].

2. El mantenimiento de la vibrante ocupa el segundo lugar, con un $22,4 \%$.

3. En tercer lugar, aparece la realización de un sonido compuesto de aspiración y [r]: $15,8 \%$. Sigue muy de cerca la pronunciación [nn] de [rn], con el $14,7 \%$.

4. La realización $\left[\begin{array}{l}1 \\ I\end{array}\right]$, con el $3,4 \%$

5. A mucha distancia, la articulación de un sonido compuesto entre aspiración y [l], con un $0,2 \%$.

2.23.2. En los Textos, han aparecido 50 casos, distribuidos del siguiente modo: 43 en interior de palabra, y 7 en posición final.

1. En interior de palabra, el $42 \%$ de los casos mantiene la vibrante; se realiza como aspirada en el $37 \%$, y como [n], dando lugar a la secuencia [nn], en el $21 \%$.

2. En posición final, se mantiene la vibrante en 2 casos, se pierde en 3 , y con 1 caso cada uno, se realiza como [n] y como [-1] ${ }^{19}$.

\subsection{Secuencia heterosilábica [-rl-]}

2.24.1. En el ALEA. Número de mapas: 2: garlopa (mapa n. ${ }^{\circ} 1583$ ), mirlo (mapa $\mathrm{n}^{\circ}{ }^{\mathrm{1}}$ 1584). El número de casos asciende a 404.

${ }^{19}$ En los textos, han aparecido 7 casos de la secuencia [ln]: 1 en posición interior de pa-

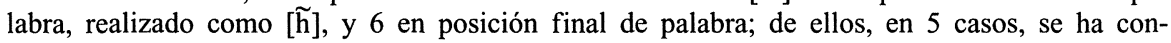
servado $[-1]$, y en otro, se ha realizado como [-r]. 


\begin{tabular}{lllllllll}
\hline & $-\mathrm{rl}-$ & $-1 \mathrm{l}-$ & $-\frac{1}{\mathrm{I}} \mathrm{l}-$ & $-\mathrm{li}-$ & $-\varnothing \mathrm{l}-$ & $-\overline{\mathrm{r}}-$ & $-\mathrm{hl}-$ & $-{ }_{\mathrm{h}}^{\mathrm{h}} \mathrm{l}-$ \\
\hline $\mathrm{Al}$ & 5 & 55 & 17,5 & & 2,5 & & 20 & \\
$\mathrm{Gr}$ & 7 & 26 & 6 & 1 & 5 & 8,2 & 40,5 & 6 \\
$\mathrm{~J}$ & 7,4 & 39 & 7,4 & 7,4 & 2 & 9 & 22,4 & 5,4 \\
$\mathrm{Co}$ & 1,8 & 35,5 & 7,5 & & & 1,8 & 50,9 & 1,8 \\
$\mathrm{Ma}$ & 2 & 48 & 4 & & 4 & & 39,5 & 2 \\
$\mathrm{Se}$ & 4 & 29,5 & 5,8 & & 5,8 & & 50,9 & 4 \\
$\mathrm{Ca}$ & 9 & 30 & 6 & & & & 55 & \\
$\mathrm{Hu}$ & 17 & 39 & 2,5 & & 2,5 & 9,7 & 29,2 & \\
And. & 6,5 & 36,6 & 7 & 1,2 & 3 & 4 & 38,2 & 3 \\
\hline
\end{tabular}

1. La realización más frecuente de [-r] es la de una aspirada, [h], en un $38,2 \%$ de los casos; esta [h] va seguida de [1] o de la geminación [11] (en este último caso, aparece con una frecuencia relativamente alta: el 16,3\%).

2. En segundo lugar, encontramos la realización de la vibrante como [1], con un $36,6 \%$; en este caso, la secuencia heterosilábica [-rl-] se convierte en la secuencia geminada [-11-].

3. Con porcentaje muy inferior a los anteriores aparece la pronunciación del sonido intermedio $\left[\begin{array}{l}1 \\ 1\end{array}\right]$, con el $7 \%$, y el mantenimiento de $[\mathrm{r}]$, con el $6,5 \%$.

4. La realización de la mencionada secuencia [-rl-] como la vibrante múltiple [ír], aparece con el $4 \%$.

5. La articulación de la [-r] como el sonido compuesto por aspiración y [r] tiene el $3 \%$. Con el mismo porcentaje, aparece la pérdida de la vibrante.

6. Como caso especial, posiblemente por metátesis de las dos consonantes líquidas, aparece [1ㅍ] , con un $1,2 \%$.

2.24.2. En los Textos, hemos encontrado 242 casos, repartidos del siguiente modo: 118 en interior de palabra, y 124 en final. Los resultados son los siguientes:

1. En interior de palabra, la vibrante se realiza como [-1] en un $57,6 \%$ de los casos, originando la secuencia geminada [-11-]. Se pierde en el 25,4\% de los casos; es aspirada en un 9,3\%; se mantiene como [-r] sólo en un $6 \%$, y como $\left[-\frac{1}{1}\right]$ en el $1,6 \%$.

2. En posición final de palabra, se pierde en el $57 \%$ de los casos; se mantiene como [-r] en un 20,2\%; como [-1], en el $14,5 \%$; como [- $\left.\frac{1}{I}\right]$, en el $4,8 \%$, y como [-h], en un $3,4 \%$.

\subsection{Secuencia heterosilábica [-lī-]}

Sólo en los Textos, donde hemos encontrado 26 casos: 6 en interior de palabra, y 20 en posición final. 
1. En posición interior de palabra, se ha mantenido la lateral en 3 casos; se ha perdido en 2, y se ha realizado como [-x] en 1 .

2. En posición final de palabra, se ha articulado como vibrante, generalmente [-I], en el $60 \%$ de los casos. Se ha mantenido como [-1] en un $30 \%$, y se ha perdido en el resto.

\subsection{Secuencia heterosilábica $[-l / v-]$}

Sólo en los Textos. Cuando en interior de palabra una [1] precede a una vocal, se silabica con ella, formando su margen prenuclear, pero la misma secuencia [lv] puede dar otros resultados cuando la lateral se encuentra en final de palabra y seguida de una vocal, sin que medie pausa entre ellas. En nuestros Textos, hemos encontrado 119 casos. En el 89\% de ellos, se ha mantenido la lateral; en el $8,5 \%$ se ha perdido, y en el $2,5 \%$ se ha realizado como [-r].

\subsection{Secuencia heterosilábica [-r/v-]}

Sólo en los Textos. Las mismas consideraciones que hacíamos en el anterior $\S 2.26$. son válidas para [r]. En nuestros Textos, hemos encontrado 148 casos de [-r] final de palabra seguida de una vocal. Las soluciones han sido las siguientes: en el $44 \%$, se ha perdido la vibrante; en el $42 \%$, se ha mantenido. Se ha pronunciado como [1] en el $12,8 \%$ de los casos. El 1,2\% restante se lo reparten, a partes iguales, entre $\left[\begin{array}{l}1 \\ \mathrm{l}\end{array}\right]$ y $[\mathrm{h}]$.

\section{CONCLUSIONES}

Del análisis de los datos allegados en este trabajo, podemos extraer las siguientes conclusiones generales:

3.1. El número de realizaciones que pueden aparecer en Andalucía como resultado de la neutralización de las consonantes $/-1 /, /-R /$ en posición implosiva es muy elevado: las más frecuentes son: $[\mathrm{r}] \mathrm{o}[\mathrm{I}]$, [1], la mixta $\left[\begin{array}{l}1 \\ 1\end{array}\right]$ y [Ø], y a mucha distancia, en cuanto a frecuencia se refiere, [h] oral o nasal, [n], las realizaciones de ambas líquidas acompañadas de aspiración, la asimilación al lugar de articulación de la consonante siguiente, originando secuencias como $[\mathrm{tt}],[\mathrm{kk}],[\mathrm{gg}],[\mathrm{ff}],[\mathrm{mm}],[\mathrm{nn}],[11]$, etc, donde la primera consonante, implosiva, suele tener poca tensión; la vocalización en [i]; la palatal lateral [1], etc. 


\section{2. [-r], [-l] en posición final de palabra}

3.2.1. Cuando las mencionadas consonantes se encuentran en posición final de palabra ante pausa, tanto en el ALEA como en los Textos, se pierden con muchísima más frecuencia en la Andalucía occidental (Ma, Se, $\mathrm{Ca}, \mathrm{Hu}$ ) que en la oriental. En el total de la región, los porcentajes de pérdida son los siguientes: en el ALEA: [-r]: 64,2\%; [-1]: 67,6\%. En los Textos: [-r]: 77,6\%; [-1]: $76 \%$. Cuando se mantienen, tanto en el $A L E A$, como en los Textos, la realización más frecuente es, para ambas, $[-1]$, seguida de [-r]; a mayor distancia $\left[\begin{array}{l}1 \\ \mathrm{I}\end{array}\right] \mathrm{y}$, luego, otras articulaciones minoritarias, como $[\mathrm{h}],[\mathrm{n}]$, [s], etc.

3.2.2. En los Textos, al tratarse de habla continua, ocurre frecuentemente que las consonantes líquidas aparecen al final de una palabra que precede a otra que comienza por vocal, sin que entre ellas medie pausa. Las soluciones en este caso son muy diferentes: a) [-r] se pierde en el $44 \%$ de los casos; se conserva en el $42 \%$; es [-1] en el $12,8 \%$, y, en el resto, $\left[-\frac{1}{d}\right]$ o [-h]. b) [-1] se conserva en el $89 \%$ de los casos; a mucha distancia ocurren su pérdida: $8,5 \%$ y la articulación $[-r]$, con el $2,5 \%$.

\subsection{Secuencia $\ll-r+$ consonante-»}

3.3.1. Cuando [-r] precede a los fonemas $/ \mathrm{p} /, / \mathrm{b} /, / \mathrm{t} /, / \mathrm{d} /, / \mathrm{k} /, / \mathrm{g} /$, las soluciones son las siguientes: a) su conservación en porcentajes muy elevados: en el $A L E A$, entre el $86,5 \%$ para [rb], y el $99,3 \%$ para [rt]. En los Textos, en posición interior, también se conserva en los mismos términos: entre el $87,5 \%$ para [rk] y el $99 \%$ para [rd]. b) En segundo lugar y, lógicamente, después de su realización como [r], en el $A L E A$, siempre aparece, a mucha distancia, [1], y, luego, otras soluciones minoritarias. En los Textos, en posición interior, las cosas ya no son tan sistemáticas: ante $/ \mathrm{b} /, / \mathrm{d} /, / \mathrm{g} /$, es $[-1]$ la realización que aparece con mayor frecuencia. Ante las oclusivas sordas, aparece cualquier otra realización.

Cuando en los Textos [-r] está en posición final de palabra precediendo a cualquiera de los fonemas oclusivos, lo más frecuente es que se pierda, siguiendo, en orden descendiente de frecuencias, su conservación, su realización como [1], y, luego, como $\left[\begin{array}{l}1 \\ 1\end{array}\right]$.

3.3.2. Cuando $[-\mathrm{r}]$ precede a los fonemas fricativos $/ \theta /, / \mathrm{s} /, / \mathrm{x} /$, las soluciones son: a) en el $A L E A$, tiende a conservarse, con porcentajes muy desiguales ante $/ \theta /(97,5 \%)$ y ante $/ \mathrm{x} /(39,7 \%)$, y a perderse ante $/ \mathrm{s} /$. b) En segundo lugar, se pierde ante $/ \theta / \mathrm{y} / \mathrm{x} / \mathrm{y}$ se conserva ante $/ \mathrm{s} /$. 
En los Textos: a) en posición interior de palabra, se conserva mayoritariamente. $b$ ) En final de palabra, tiende a perderse.

3.3.3. La vibrante $[-\mathrm{r}]$, en los Textos, se conserva siempre ante $/ \hat{\mathrm{c}} /$, en cualquier posición.

3.3.4. Cuando [-r] precede a los fonemas nasales, las soluciones son muy diferentes:

a) En la secuencia [-rm-], la vibrante se conserva en un número muy elevado de casos: en el $A L E A$, en un 80,4\%; en los Textos, en posición interior de palabra, en un $98 \%$; la segunda realización es, en ambos casos, a mucha distancia, [- $\left.{ }_{1}^{1}\right]$ y [-1] en el Atlas, y [-1] en los Textos. En la primera obra, siguen otras realizaciones, como aspiración nasal, [m], vibrante aspirada, etc.

En los Textos, en posición final de palabra, se conserva en el $50 \%$ de los casos, y se pierde en el otro $50 \%$.

b) En la secuencia [-rn-], los resultados están más dispersos: en el $A L E A$, la primera realización es una aspirada nasal, seguida de la conservación de $[-r]$, de una vibrante aspirada, de $\left[-\frac{1}{I}\right]$, etc. En los Textos, en posición interior, se conserva, en primer lugar, después se aspira, y, por último, se realiza como [n], dando lugar a la secuencia [nn].

En los Textos, en posición final de palabra, hay muy pocos casos; las realizaciones de [-r] han seguido este orden: pérdida, conservación, y realización como [1] o como [n].

3.3.5. En la secuencia [-rl-], coinciden el $A L E A$ y los Textos, en posición interior, donde la conservación de [-r] ocupa una frecuencia muy baja: el $6,5 \%$ y el $6 \%$, respectivamente. En la primera obra, la articulación más frecuente es $[-\mathrm{h}](38,2 \%)$, seguida de $[-1](36,6 \%)$, de $\left[-\frac{1}{1}\right]$ ( $\left.7 \%\right)$, etc.; en la segunda, el orden es: [-1], su pérdida, [-h], etc.

En los Textos, en posición final, se pierde en primer lugar; luego, se conserva, se pronuncia como [1], sigue $\left[\begin{array}{l}1 \\ \mathrm{I}\end{array}\right]$ y $[\mathrm{h}]$.

\subsection{Secuencia «-l+consonante»}

3.4.1. Cuando [-1] precede a los fonemas $/ \mathrm{p} /, / \mathrm{b} /, / \mathrm{t} /, / \mathrm{d} /, / \mathrm{k} /, / \mathrm{g} /$, se realiza en la mayoría de los casos como [-r]. En el $A L E A$, ante los fonemas. dentales y velares, el porcentaje más alto corresponde a su articulación como vibrante: los porcentajes van desde el $64,6 \%$ en [rk] hasta el $80,3 \%$ en [rg]. En segundo lugar, y a bastante distancia, se mantiene la [-1], con por- 
centajes entre el $11,2 \%$ para [lg] y el $20,1 \%$ para [ $1 \mathrm{k}]$; en todos los casos, aparece en tercer lugar la realización mixta $\left[\begin{array}{l}1 \\ 1\end{array}\right]^{20}$. Ante $/ \mathrm{b} /$, también es $[-\mathrm{r}]$ la realización más frecuente, con el $40 \%$, seguida a corta distancia de [-1] (31\%) y, luego, su pérdida y la articulación $\left[\begin{array}{l}1 \\ 1\end{array}\right]$. Ante $/ \mathrm{p} /$, las cosas cambian: el primer puesto lo ocupa la pérdida de la lateral con el $39 \%$; en segundo lugar, $[-\mathrm{r}](35 \%)$, y luego, $[-1]$ y $\left[-\frac{1}{1}\right]$.

En los Textos, en posición interior de palabra, también es [-r] la manifestación más frecuente ante todos los fonemas oclusivos, con frecuencias comprendidas entre el $50 \%$ para [rd] y el $75 \%$ para [rp]; en segundo lugar, y en general, también se mantiene $[-1]^{21}$, con porcentajes entre el $20 \%$ para $[1 \mathrm{k}]$ y el $42 \%$ para [ld].

En los Textos, en posición final de palabra, la lateral se mantiene ante todas las consonantes, excepto / $\mathrm{t} /$, donde es [rt]; los porcentajes se extienden desde el $47,5 \%$ en [lp] hasta el $72 \%$ en [ld]; la segunda realización suele ser $[-r]$, y la tercera, $\left[\begin{array}{l}1 \\ l\end{array}\right]$ o su desaparición.

3.4.2. Cuando [-1] precede a fonemas fricativos, en el $A L E A$, se realiza en primer lugar como [-r] con porcentajes entre el $61,4 \%$ en [rf] y el $84,4 \%$ en $[\mathrm{r} \theta]$; siguen, a mucha distancia, $[-1]$, y luego $\left[-\frac{1}{1}\right]$; otras soluciones son muy minoritarias.

En los Textos, en posición interior de palabra, y con un número muy desigual de realizaciones, hemos encontrado predominio de articulación de la lateral en [lf], mientras que ante $[\theta]$, es [-r].

En los mismos Textos, en posición final de palabra, las realizaciones de [-1] son distintas: se mantiene en [10] $(62,5 \%)$, pero es vibrante en [rf] $(66,6 \%)$; la segunda solución es, en ambos casos, la otra líquida, y, por último, su pérdida.

3.4.3. La lateral [-1] ante [ĉc], en el $A L E A$, es [-r] en el $79,6 \%$ de los casos; siguen $[-1](12,1 \%)$ y $\left[-\frac{1}{d}\right](8,2 \%)$.

3.4.4. En la secuencia [-lm-], el comportamiento ha sido muy diferente entre el $A L E A$ y los Textos. En la primera obra, el mantenimiento de [-1] $(32,2 \%)$ y su realización como [-r] $(32,3 \%)$ es lo más frecuente, con escasas diferencias; siguen su pérdida, su realización como $\left[-\frac{1}{1}\right]$ y otros sonidos con pocas ocurrencias.

${ }^{20} \mathrm{Y}$ luego, otras realizaciones minoritarias como las siguientes, en orden descendente de frecuencia: [-h], asimilación a la consonante siguiente en [kk] y [tt], vibrante aspirada, pérdi$\mathrm{da},[\mathrm{i}]$.

${ }^{21}$ Con la excepción de $/ \mathrm{lb} /$, donde el segundo lugar es para $\left[\begin{array}{l}1 \\ 1\end{array}\right]$, y el tercero para [1]. 
En los Textos, en posición interior de palabra, predomina la vibrante $(58 \%)$; en segundo lugar, la articulación mixta $\left[-\frac{1}{I}\right](26 \%)$, y en último, $[-1]$ $(16 \%)$.

En los Textos, en posición final de palabra, predomina también [-r] (62\%); sigue [-1] (33\%), y con una frecuencia muy baja, $\left[-\frac{1}{I}\right]$ y su pérdida.

3.4.5. No tenemos datos contabilizables de la secuencia $/ \mathbf{l} /$ en el $A L E A$, y los que han aparecido en los Textos son muy escasos, y las soluciones, en cualquier posición, muy diversas: $[-1],[-1]$, su pérdida, etc.

3.4.6. De lo anteriormente expuesto, se deduce que, cuando las consonantes líquidas estudiadas se encuentran en posición final de palabra, ante pausa, o en secuencias heterosilábicas en interior de palabra, sus realizaciones son idénticas en el $A L E A$ y en los Textos en lo que se refiere al orden de frecuencia de sus soluciones ${ }^{22}$. Sólo en las secuencias [-rn-], [-rl-], [-lp-] y [-1f-] no se produce esta coincidencia ${ }^{23}$.

\section{EXPLICACIÓN DE ESTOS CAMBIOS}

¿Cómo se pueden explicar estos cambios?

Los fonemas $/ 1 / \mathrm{y} / \mathrm{r} /$ tienen muchos rasgos comunes: son líquidos $-\mathrm{y}$, por lo tanto, poseen los rasgos consonántico, vocálico, sonoro-, apicoalveolares y se articulan con la lengua más o menos cóncava, formando un resonador bucal amplio. Entre ellos se diferencian porque /1/ es continuo y $/ \mathrm{r} /$, interrupto.

Examinaremos primero los cambios más frecuentes, que son, como hemos visto $[\mathrm{r}]>[1],[1]>[\mathrm{r}]$ y la evolución de [1] o [r] a la consonante mixta $\left[\begin{array}{l}1 \\ 1\end{array}\right]$.

4.1. La causa del cambio [r] > [1] se explica fácilmente como un fenómeno de lenición o relajación articulatoria. Según Straka ${ }^{24}$, la falta de tensión articulatoria se refleja sobre todo en los bordes laterales de la lengua

\footnotetext{
${ }^{22}$ Por ejemplo, en la secuencia [-r $\left.\theta-\right]$, el rango de las soluciones en el $A L E A$ y en los Textos es el siguiente: $1 .^{\circ}:[\mathrm{r}] ; 2 .^{\circ}:[\varnothing] ; 3 .^{\circ}:[1]$.

${ }^{23}$ Coinciden en ambas fuentes: [-1], [-r] finales ante pausa, y las secuencias [rp], [rb], [rt], [rd], [rk], [rg], [r $\theta],[\mathrm{rs}],[\mathrm{rx}],[\mathrm{rm}],[\mathrm{lb}],[\mathrm{lt}],[\mathrm{ld}],[\mathrm{lk}],[\mathrm{lg}]$ y $[1 \theta]$.

${ }_{24}$ Georges Straka, "Contribution a l'histoire de la consonne $r$ en français», Neuphilologische Mitteillungen, 66, 1965, págs. 572-606. Recogido en: Georges Straka, Les sons et les mots. Choix d'études de phonétique et de linguistique, Paris, Klincksieck, 1979, págs. 464499. La referencia, en la pág. 482.
} 
que se separan de los bordes laterales de la bóveda del paladar y de los molares, mientras que, simultáneamente, la punta de la lengua, que ha perdido su elasticidad y ya no vibra, busca un punto de apoyo, que será la zona de los alvéolos sobre la que antes producían las vibraciones. De este modo, pasa de interrupta a continua.

En el sonograma de [pára] para de la figura 1, se puede percibir claramente la interrupción o pequeña oclusión que supone la vibración de la $[\mathrm{r}]$ simple. Si esa oclusión desaparece, por el fenómeno de la lenición antes mencionado, esta líquida interrupta se convierte en la líquida continua [1], dando origen a [pála] pala, como muestra el sonograma de la figura 2 .

¿Cómo pueden aparecer tan claros esos formantes de [1] que casi se confunden con los de las vocales [a] vecinas?

En el sonograma de [pára] parra de la figura 3, la vibrante es múltiple: tiene tres oclusiones breves, señaladas por «O» en la figura; entre ellas, hay dos breves elementos vocálicos, señalados por «E», que son los que aparecen en el momento en que el ápice de la lengua se separa de los alvéolos para dar paso a la oclusión siguiente. Si las oclusiones desapareciesen, quedarían los formantes de los elementos «E», que serían una continuación entre las dos [a], semejante a la [1] de [pála] de la figura 2 .

La misma explicación es válida para el cambio de [-r] a [-1]. En la figura 4, tenemos el sonograma de [márta] Marta, con la secuencia [-rt-], donde [-r] es implosiva. Después de la [á], aparece la breve oclusión de esta vibrante, seguida del elemento esvarabático. Si la oclusión desaparece, la interrupta se convierte en continua, dando origen a un espectro semejante al de [málta] malta, de la figura 5.

4.2. El cambio [1] $>[\mathrm{r}]^{25}$, que conlleva el paso de una continua a una interrupta es más difícil de explicar. Según el mismo Straka ${ }^{26}$, al perder el ápice o punta de la lengua contacto con los alvéolos, se adhiere a ellos muy ligeramente y puede, bajo la presión del aire espirado, iniciar una vibración, y comenzar a articular así una [r] apical ${ }^{27}$.

\footnotetext{
${ }^{25}$ Otros cambios de /// que aquí no tratamos, pero que se deben al mismo fenómeno articulatorio de la lenición, son: a) la vocalización de $-l$ en $u$ : $\mathrm{s}$ a $1 \mathrm{t} \mathrm{u}>$ sauto $>$ soto; recordemos que la $l$ latina implosiva ante consonante era «pinguis» o velarizada; al despegarse el ápice de la lengua de los alvéolos y descender, se eleva el postdorso de la lengua, hasta que el contacto lingual anterior se sustituye por la posición posterior, dando la $u$.

${ }^{26}$ Vid. Georges Straka, "Contribution à la description et à l'histoire des consonnes L», Travaux de Linguistique et de Littérature, 6, 1968, págs. 267-326. Recogido en: Georges Straka, Les sons et les mots, págs. 364-421. La referencia, en la pág. 404.

${ }^{27}$ Léonce Roudet, al intentar justificar este cambio en sus Eléments de Phonétique générale (París, 1910, págs. 303-304), dice que para que $l$ y $n$ puedan pasar a $r$ basta «una modificación muy débil en los movimientos orgánicos».
} 


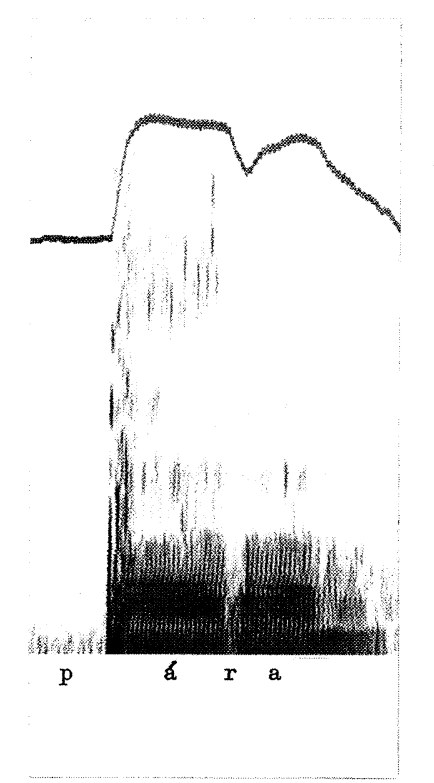

Fig. 1

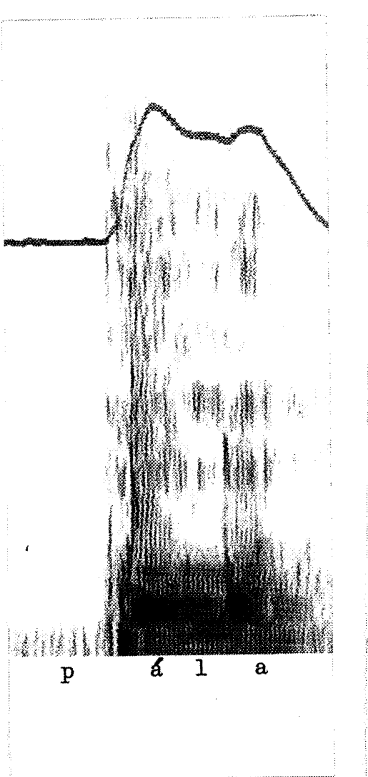

Fig. 2

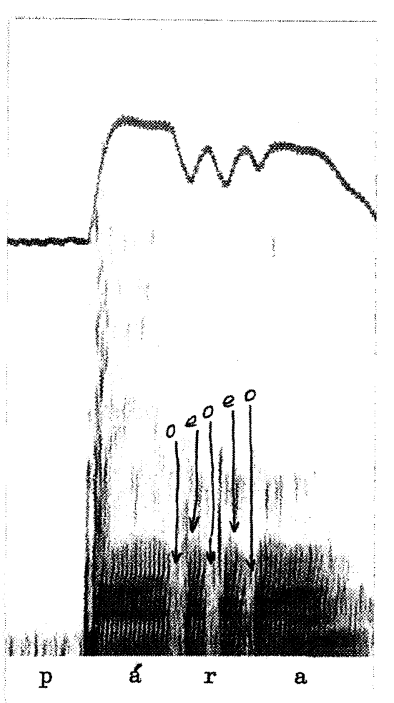

Fig. 3

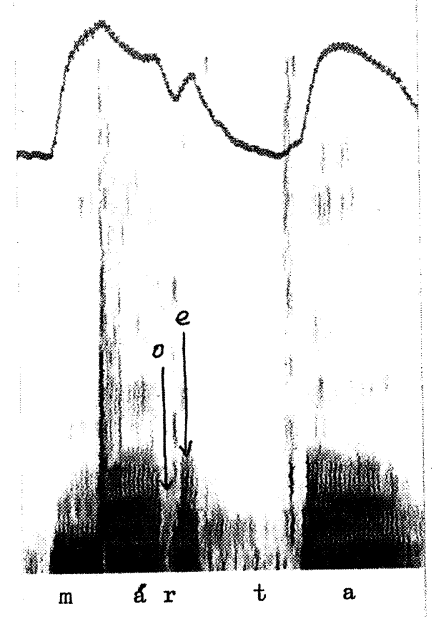

Fig. 4

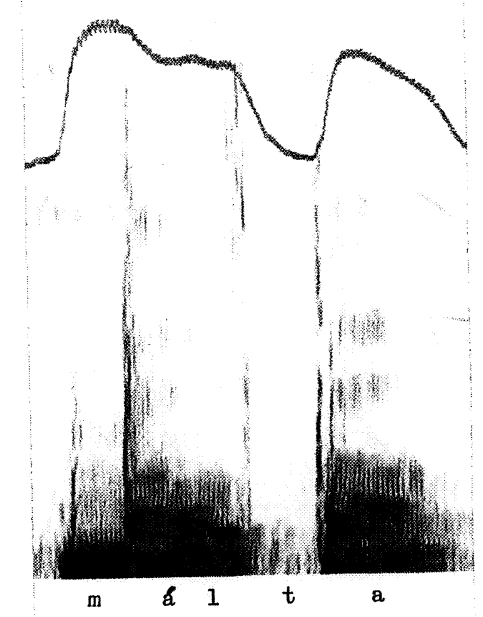

Fig. 5 
4.3. La pérdida de [-1] y [-r] implosivas se explica, como indica Léonce Roudet ${ }^{28}$ por un relajamiento progresivo de la energía articulatoria y respiratoria, relajamiento que se une a la ya débil posición articulatoria de la parte distensiva de la sílaba en español, como pusieron de manifiesto, sobre todo, Amado Alonso ${ }^{29}$ y Bertil Malmberg ${ }^{30}$.

\subsection{La misma lenición articulatoria explica los demás cambios}

4.4.1. La aparición de una consonante nasal, [n] o [m], según la consonante siguiente, $o[\eta]$ en posición final ante pausa, implica el descenso del velo del paladar, manteniéndose cerrada la articulación anterior. Este cambio aparece en muchas secuencias consonánticas, pero con una frecuencia muy pequeña.

4.4.2. Si se pierde el contacto del ápice de la lengua sobre los alvéolos, el descenso de la parte anterior de la lengua puede llevar consigo la aproximación del postdorso o de la raíz de la lengua al velo del paladar o a la pared faríngea, en cuyo caso, se sustituye la articulación anterior por una posterior: la aspirada [h]. Es un caso paralelo al de la aspiración de la [-s] implosiva, que tan claramente explicó Josef Chlumsky ${ }^{31}$. Este cambio aparece también en muchas ocasiones pero con una frecuencia muy baja.

4.4.3. Como paso previo al caso anterior, puede producirse una articulación mixta en la que aún se mantenga el contacto anterior y se esté produciendo, al mismo tiempo, el posterior. Son los casos de $\left[\begin{array}{l}\mathrm{h} \\ \mathrm{h}\end{array}\right]$, vibrante aspirada; $\left[\begin{array}{l}\mathrm{h} \\ 1\end{array}\right]$, lateral aspirada, etc., que no aparecen muchas veces y, cuando lo hacen, tienen una frecuencia muy baja.

4.4.4. Los casos de vocalización siempre han aparecido cuando [1] o [r] preceden a las velares $[\mathrm{k}] \mathrm{o}[\mathrm{g}]$ : la pérdida de contacto anterior de la lengua, su cambio desde la posición cóncava a la convexa, favorecido por la elevación del postdorso para la articulación de las siguientes consonantes velares, da origen a una posición lingual que produce [i].

\footnotetext{
${ }^{28}$ Op. cit., pág. 305.

29 «Una ley fonológica del español», Hispanic Review, XIII, 1945, págs. 91-101. Recogido en sus Estudios Lingüisticos. Temas españoles, Madrid, Ed. Gredos, 1954, págs. 288-307.

${ }^{30}$ «La structure syllabique de l'espagnol. Étude de phonétique», Boletim de Filología (Lisboa), IX, 1948, págs. 99-120. Recogido en sus Estudios de Fonética Hispánica, Madrid, C.S.I.C., 1965, págs. 3-28.

${ }^{31}$ «L's andalouse et le sort de l's indo-européenne finale en eslave», Slavia (Praga), VII. 1928-29, págs. 750-753. Traducido al español en Publicaciones del ALEA, Tomo III, núm. 2, Granada.
} 
4.4.5. Otro cambio es: $[\mathrm{r}]>[\mathrm{s}]{ }^{32}$; en este caso, desaparece la oclusión de la vibrante, y se ensordece.

4.4.6. La aparición de las consonantes geminadas [tt] $(<[\mathrm{lt}])$, [gg] $(<[\mathrm{rg}]),[\mathrm{kk}](<[\mathrm{lk}]),[\mathrm{ff}](<[\mathrm{lf}]),[\mathrm{mm}](<[\mathrm{rm}],[\mathrm{lm}]),[\mathrm{nn}](<[\mathrm{rn}])$ se debe a que, perdida la tensión articulatoria de la consonante implosiva, ésta se asimila al lugar de articulación de la consonante prenuclear siguiente, y al no querer el hablante perder el segmento implosivo, mantiene el nuevo asimilado. Estos casos, aunque relativamente numerosos, tienen una frecuencia de ocurrencias baja.

4.4.7. El último cambio es el de [-rl-] $>[-\overline{\mathrm{r}}-]$ (§ 2.24.). De los dos casos, examinados, garlopa y mirlo, el fenómeno se da sólo en este último, en cuatro provincias, y con una frecuencia no despreciable: el $4 \%$. Su explicación es la siguiente: en garlopa, la [a] de la sílaba [gar-] es una vocal baja, central; cuando se articula la [-r], el ápice de la lengua sube y este órgano adopta una forma más o menos cóncava; al cesar la pronunciación de la vibrante, se debe articular la [1], para lo que fácilmente se mantiene la articulación apicoalveolar y el borde o los bordes de la lengua se separan de las encías laterales y de los molares para dar paso al aire de la lateral. En mirlo, la [í] es una vocal alta anterior; cuando se va a pronunciar la [-r], el dorso y el predorso linguales tienen que descender para dejar el espacio suficiente con el fin de permitir que el ápice de la lengua se eleve y realice la vibración o las vibraciones de [-r]; la forma de la lengua en la posición inmediatamente anterior a la vibrante es convexa, por lo que fácilmente pueden quedar adheridos los bordes de la lengua a las encías y dar como resultado una [ī] múltiple, en lugar de [-r+l-].

\footnotetext{
${ }^{32}$ Es el cambio que empezó a producirse en una época en francés y dejó el doblete chaire "cátedra» y chaise «silla», o el paso bericle > besicles «quevedos», "antiparras».
} 